\title{
Fibrin(ogen) exacerbates inflammatory joint disease through a mechanism linked to the integrin $\alpha_{M} \beta_{2}$ binding motif
}

\author{
Matthew J. Flick, Christine M. LaJeunesse, Kathryn E. Talmage, David P. Witte, \\ Joseph S. Palumbo, Malinda D. Pinkerton, Sherry Thornton, and Jay L. Degen \\ Cincinnati Children's Hospital Research Foundation and University of Cincinnati College of Medicine, Cincinnati, Ohio, USA.
}

\begin{abstract}
Fibrin deposition within joints is a prominent feature of arthritis, but the precise contribution of fibrin(ogen) to inflammatory events that cause debilitating joint damage remains unknown. To determine the importance of fibrin(ogen) in arthritis, gene-targeted mice either deficient in fibrinogen ( $\mathrm{Fib}^{-}$) or expressing mutant forms of fibrinogen, lacking the leukocyte receptor integrin $\alpha_{M} \beta_{2}$ binding motif $\left(F i b \gamma^{390-396 A}\right)$ or the $\alpha_{I I b} \beta_{3}$ platelet integrin-binding motif (Fib $\gamma^{\Delta 5}$ ), were challenged with collagen-induced arthritis (CIA). Fib- mice exhibited fewer affected joints and reduced disease severity relative to controls. Similarly, diminished arthritis was observed in Fib $\gamma^{390-396 A}$ mice, which retain full clotting function. In contrast, arthritis in Fib $\gamma^{\Delta 5}$ mice was indistinguishable from that of controls. Fibrin(ogen) was not essential for leukocyte trafficking to joints, but appeared to be involved in leukocyte activation events. $\mathrm{Fib}^{-}$and $\mathrm{Fib} \gamma^{390-396 \mathrm{~A}}$ mice with CIA displayed reduced local expression of TNF- $\alpha$, IL-1 $\beta$, and IL-6, which suggests that $\alpha_{M} \beta_{2}$-mediated leukocyte engagement of fibrin is mechanistically upstream of the production of proinflammatory mediators. Supporting this hypothesis, arthritic disease driven by exuberant TNF- $\alpha$ expression was not impeded by fibrinogen deficiency. Thus, fibrin(ogen) is an important, but context-dependent, determinant of arthritis, and one mechanism linking fibrin(ogen) to joint disease is coupled to $\alpha_{M} \beta_{2}$-mediated inflammatory processes.
\end{abstract}

\section{Introduction}

Rheumatoid arthritis (RA) is a common and debilitating disease involving synovial hyperplasia, chronic inflammation, edema, tissue degeneration, local fibrin deposition and dissolution, progressive loss of cartilage and bone, and ultimately loss of joint mobility and function $(1,2)$. While the precise etiology of RA is not fully defined, 2 complementary immunological processes appear to participate early in disease pathogenesis. Primary or secondary activation of the adaptive immune system, particularly $\mathrm{T}$ cells expressing primarily Th1 cytokines and autoantibody-producing B cells, seems to play a major role in disease onset and/or early progression (1-4). In addition, cytokine-driven processes leading to the infiltration and activation of neutrophils, macrophages, and other inflammatory cells within joints are an early, if not causative, event in the development of degenerative inflammatory joint disease. The local elaboration and balance between pro- and anti-inflammatory factors appear to be major determinants of arthritis progression in joints of both human subjects and experimental animals $(2,3,5,6)$.

Among the powerful immune modulators, TNF- $\alpha$ is pivotal. TNF- $\alpha$ directly controls the functional properties and survival of leukocytes and other cells and regulates the expression of many key cytokines $(1,3,5)$. The clinical significance of TNF- $\alpha$ is highlighted by the fact that neutralizing agents against TNF- $\alpha$ are widely, although not universally, effective in the treatment of RA and experimental inflammatory arthritis $(7,8)$. Despite the overwhelming evidence implicating TNF- $\alpha$ (and other cytokines) in

Nonstandard abbreviations used: AIA, antigen-induced arthritis; CIA, collageninduced arthritis; CII, type II collagen; MPO, myeloperoxidase.

Conflict of interest: The authors have declared that no conflict of interest exists. Citation for this article: J. Clin. Invest. 117:3224-3235 (2007). doi:10.1172/JCI30134. the progression of inflammatory joint disease, the exact regulatory mechanisms leading to the local production of these factors have yet to be elucidated. However, changes in soluble and extracellular matrix-associated factors within the joint microenvironment are undoubtedly of central importance.

One local reactive change that has been consistently associated with inflammatory events is the activation of the hemostatic system. An increasingly convincing body of evidence suggests that not only is the activation of the coagulation and fibrinolytic systems coincident with the development of inflammatory processes, but that hemostatic factors actively contribute to the regulation of inflammatory processes in vivo $(7,9-14)$. The accumulation of thrombin-antithrombin complexes, fibrin, and fibrin degradation products implies that activation of the coagulation and fibrinolytic systems are ongoing phenomena within diseased joints. Thrombin is the central hemostatic protease that initiates fibrin polymerization and was previously implicated as a major participant in the pathogenesis of experimental inflammatory joint disease. The potent thrombin inhibitor hirudin was shown to reduce joint disease severity in the mouse models of antigen-induced arthritis (AIA) and collagen-induced arthritis (CIA) $(15,16)$. These findings were also correlated with both reduced fibrin deposition within joints and reduced local proinflammatory cytokine levels. In addition, agents that suppress either thrombin generation (e.g., activated protein C) or activity (e.g., antithrombin III) are reported to reduce acute and chronic inflammatory processes in vivo (17).

Fibrin deposition is one of the most conspicuous and consistent features of both human RA and experimental animal models of arthritic disease, documented in areas of synovial hemorrhage and necrosis and more broadly in areas of synovial inflammation and hyperplasia and along articular surfaces $(16,18,19)$. Furthermore, fibrin(ogen) is a key component of particulate "rice bodies" within 
A
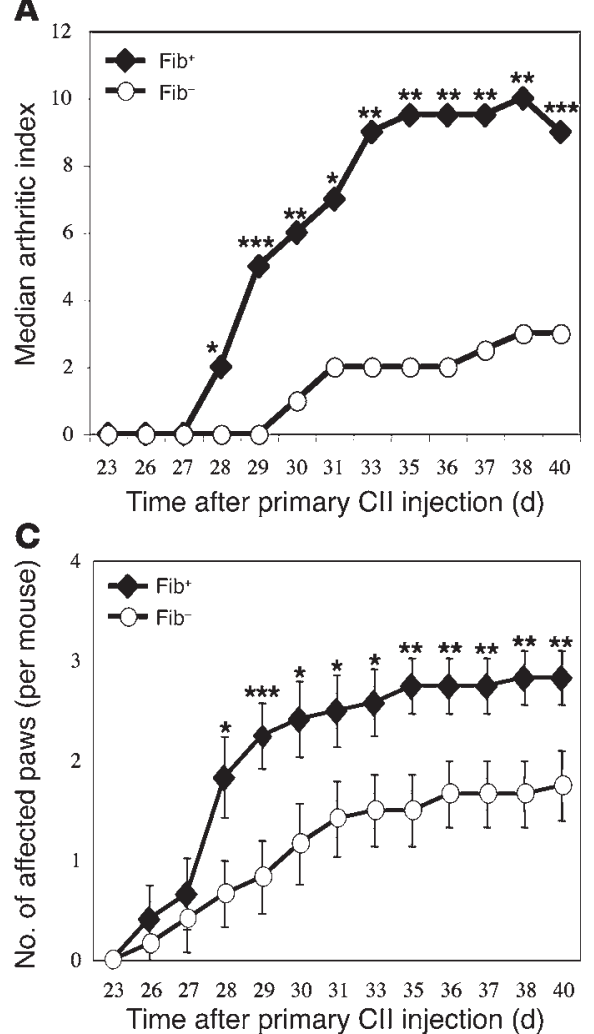

B

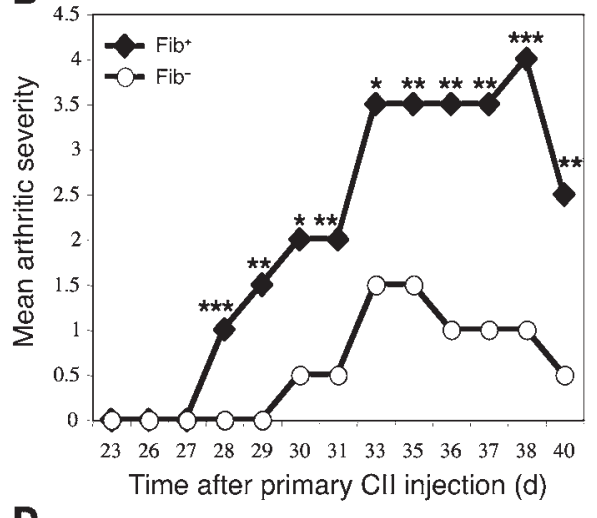

D

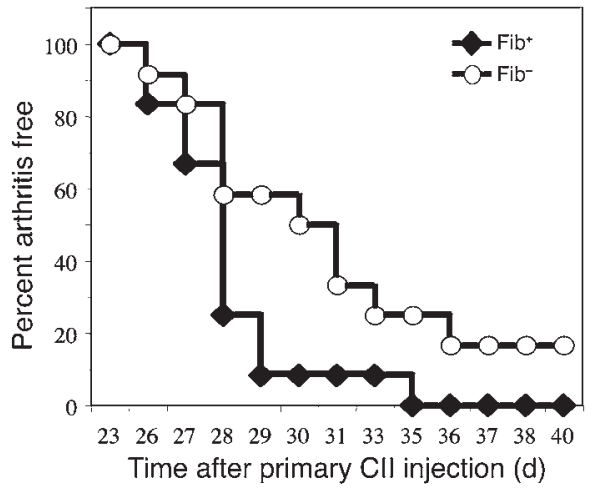

\section{Figure 1}

Fibrinogen deficiency results in diminished macroscopic CIA. Representative experiment with cohorts of male $\mathrm{Fib}^{+}$and $\mathrm{Fib}^{-}$mice ( $n=12$ per group) immunized with CII. (A) Median arthritic index. (B) Median arthritic severity of paws. ${ }^{\star} P<0.05,{ }^{\star \star} P<0.03,{ }^{\star \star \star} P<0.01$, Mann-Whitney $U$ test. (C) Number of arthritic paws per mouse. Values are mean \pm SEM. ${ }^{*} P<0.05$, ${ }^{\star *} P<0.03$, ${ }^{\star \star *} P<0.01$, Student's $t$ test. (D) Percentage of mice free of any observable macroscopic inflammatory joint disease in the fore and hind paws. $P<0.03$, Kaplan-Meier log-rank analysis. synovial fluid (18). Fibrin(ogen), a classic acute phase reactant, appears to be a key bridging molecule between the hemostatic and inflammatory systems. Not only are fibrinogen production and deposition controlled by inflammatory events, but an increasing body of evidence suggests that fibrin and/or immobilized fibrinogen regulates local inflammatory processes. Thus, fibrin(ogen)rich matrices may both support inappropriate tissue reorganization within arthritic joints and work in tandem with soluble immune modulators to support local leukocyte activation and the secondary expression of specialized functions.

To directly evaluate the importance of fibrin(ogen) in the progression of inflammatory joint disease, we used fibrinogendeficient $\left(\mathrm{Fib}^{-}\right)$mice as well as mice expressing mutant forms of fibrinogen that retain clotting function but lack selected integrin receptor binding motifs (i.e., $\alpha_{I I b} \beta_{3}$ and $\left.\alpha_{M} \beta_{2}\right)(14,20)$. Two established experimental settings of murine arthritis with distinct etiologies were used: (a) adaptive immunity-driven CIA and (b) cytokine-driven arthritis in human TNF- $\alpha$-overexpressing transgenic Tg197 mice (21). Our findings established that fibrin(ogen) can serve as a powerful determinant of inflammatory joint disease in the absence of any experimentally imposed trauma to joints. Furthermore, fibrin(ogen) is mechanistically coupled to arthritic disease through leukocyte integrin $\alpha_{M} \beta_{2}$, but not platelet integrin $\alpha_{\mathrm{II}} \beta_{3}$ engagement of fibrin(ogen). However, the importance of fibrin deposition in inflammatory joint disease is context dependent and appears to be superseded in arthritis driven by exuberant TNF- $\alpha$ expression. These findings are consistent with the emerging concept that leukocyte engagement of fibrin within challenged tissues promotes arthritic disease, at least in part, by regulating inflammatory cell function, including local cytokine production.

\section{Results}

Fibrinogen deficiency results in reduced incidence and severity of CIA. To determine the contribution of fibrin(ogen) to inflammatory joint disease development and progression, mice with genetically imposed fibrinogen deficiency were immunized with bovine type II collagen (CII). The response to $\mathrm{CII}$ immunization is dependent on multiple genetic factors, including $\mathrm{MHC}$ haplotype. To ensure a robust response in control mice based on MHC haplotype and minimize variability in response caused by strain variation, the previously characterized fibrinogen knockout allele (22) was backcrossed 6 generations to the highly CIA-susceptible DBA/1 (MHC class II $\mathrm{H}-2 \mathrm{q}$ haplotype) strain. Macroscopic evaluation of the fore and hind paws of $\mathrm{Fib}^{-}$and fibrinogen-sufficient $\left(\mathrm{Fib}^{+}\right)$control mice shortly after secondary $\mathrm{CII}$ immunization revealed an obvious distinction in the development of arthritis (Figure 1A). Fib ${ }^{-}$mice developed appreciably less joint disease, as shown by a statistically significant diminution in clinical arthritic index scores (see Methods), at each time point beginning at day 28 after primary CII injection (Figure 1A). Fib $^{-}$mice also had a statistically significant decrease in arthritic severity, as evaluated using a score based on the degree of swelling for each paw (see Methods), from day 28 to the end of the evaluation period (Figure 1B). Diminished arthritic disease in $\mathrm{Fib}^{-}$mice was also readily appreciated when the number of affected paws for each mouse was compared between genotypes. As shown in Figure 1C, Fib $^{-}$mice had statistically fewer arthritic paws over the course of the entire evaluation period, including the terminal evaluation on day 40. Although a significant fraction of Fib- mice remained free of any clinically apparent joint disease under conditions where $\mathrm{Fib}^{+}$mice uniformly developed arthritis, fibrin(ogen) was clearly not mandatory for CIA because many $\mathrm{Fib}^{-}$mice ultimately developed at least 1 affected joint (Figure 1D). Nevertheless, a statistically significant dif- 

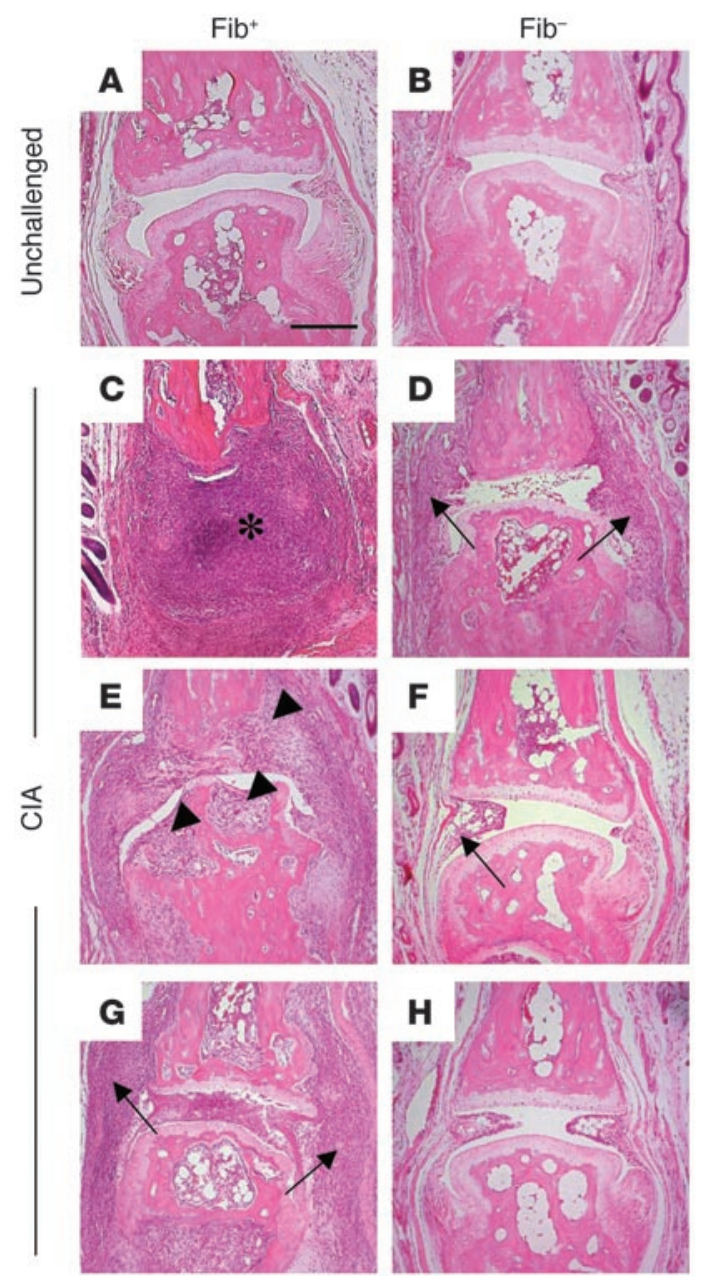
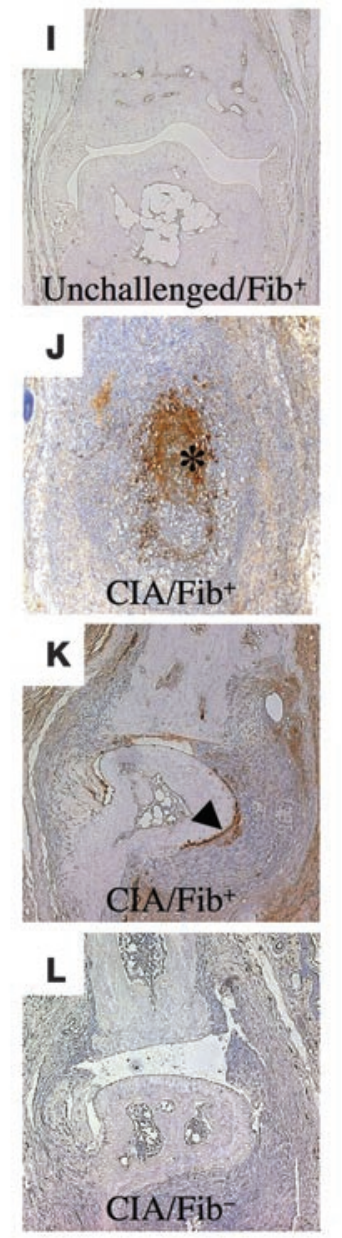

Figure 2

Microscopic analysis of the metacarpophalangeal joints of $\mathrm{Fib}^{+}$and $\mathrm{Fib}^{-}$mice immunized with $\mathrm{Cll}$. All joints were sectioned in the plane of the fore paw. (A and $\mathbf{B}$ ) Representative joint sections from unchallenged $\mathrm{Fib}^{+}$and $\mathrm{Fib}^{-}$mice. $(\mathbf{C}-\mathbf{H})$ Representative joint sections from Cll-immunized $\mathrm{Fib}^{+}$and $\mathrm{Fib}^{-}$mice harvested at day 40 and stained with hematoxylin and eosin. ClA-challenged $\mathrm{Fib}^{+}$mice generally exhibited severe joint pathology, with individuals occasionally displaying obliterated joint architecture (C) and granulation tissue invading into the underlying trabecular bone. The metacarpophalangeal joints from $\mathrm{Fib}^{+}$mice often displayed significant cartilage and bone erosion (E, arrowheads) with robust inflammatory infiltrates and synovial hyperplasia (G, arrows). Asterisk in $\mathbf{C}$ denotes pannus tissue. Joints from Cll-immunized Fib- mice typically displayed far less overall joint space damage with only mild synovial inflammation and hyperplasia ( $\mathbf{D}$ and $\mathbf{F}$, arrows). Many metacarpophalangeal joints from $\mathrm{CIA}$-challenged Fib- mice were nearly free of microscopically apparent disease (H). (I-L) Immunohistochemical stain of fibrin(ogen) within joints of unchallenged and CII-immunized mice harvested 40 days after primary CII immunization. No fibrin deposition was observed within joints from unchallenged Fib ${ }^{+}$mice (I). However, robust fibrin staining (brown, denoted by asterisk) was observed within inflamed and hyperplastic synovial tissue (J) and on articular surfaces (K, arrows) in Fib ${ }^{+}$mice. As expected, no fibrin(ogen) was detectable within joint sections prepared from Cll-immunized $\mathrm{Fib}^{-}$mice (L) regardless of the degree of joint damage. Scale bar: $100 \mu \mathrm{m}$.

ference in the course of disease development was observed between $\mathrm{Fib}^{+}$and $\mathrm{Fib}^{-}$cohorts even when the disease status of individuals was scored as simply present or absent (i.e., making no distinction between one and multiple affected joints and with no discrimination with regard to severity; Figure 1D). Multiple independent experiments of the same design using groups of all male mice gave similar results. Complementary studies of clinical arthritic index and severity focused exclusively on $\mathrm{Fib}^{+}$and $\mathrm{Fib}^{-}$female mice revealed that fibrin(ogen) was a significant determinant of joint disease regardless of mouse sex. Fib- mice developed appreciably less severe disease relative to $\mathrm{Fib}^{+}$mice (see Supplemental Figure 1; supplemental material available online with this article; doi:10.1172/JCI30134DS1).

Fibrinogen deficiency results in reduced CIA-mediated joint destruction. To ascertain whether the genotype-dependent differences in clinical evidence of arthritis were associated with differential degenerative histological changes, fore paws from $\mathrm{Fib}^{+}$ and Fib $^{-}$mice were harvested at day 40 for evaluation. Metacarpophalangeal joints from unchallenged $\mathrm{Fib}^{+}$and $\mathrm{Fib}^{-}$mice were histologically indistinguishable (Figure 2, $\mathrm{A}$ and $\mathrm{B})$. Each displayed mature intact bones with smooth surface articular cartilage, and a thin synovial membrane approximately 1-2 cell layers thick encapsulated the clear joint space, indicating that fibrinogen deficiency does not compromise normal joint development. As expected, sections of fore paws from CIIimmunized $\mathrm{Fib}^{+}$mice revealed severe joint disease characterized by robust inflammatory cell infiltrates, expansion of the joint caused by massive synovial hyperplasia and edema, and significant erosion of articular cartilage with - in some cases - complete loss of articular surfaces (Figure 2, C, E, and $\mathrm{G})$. In joints with the most severe disease, bone was eroded and replaced by invading granulation tissue with inflammatory cell infiltrates penetrating to the marrow (Figure 2, C and E). In contrast, the fore paw joints analyzed from CII-challenged Fib $^{-}$mice typically had markedly reduced joint pathology (Figure 2, D, F, and H), and individual joints were often comparable to those observed in unchallenged mice (Figure 2H). The affected Fib- joints typically only possessed limited synovial hyperplasia and modest inflammatory cell infiltrates (Figure 2, D and F). Cartilage degeneration or perturbation of the normal bone architecture was rarely observed in Fib $^{-}$mice. Fibrin deposition was absent in joints harvested from unchallenged mice (Figure 2I), but was readily apparent by immunohistochemical analysis of tissue sections in the affected joints of CII-immunized $\mathrm{Fib}^{+}$mice. Staining for fibrin was often prominent along articular surfaces exhibiting cartilage degradation, but was also observed throughout inflamed joint tissues (Figure 2, J and K). As previously reported by other investigators $(16,19)$, fibrin staining in joints from CII-immunized $\mathrm{Fib}^{+}$mice appeared to correlate with the degree of joint pathology and was less apparent in the joints of challenged mice lacking other 

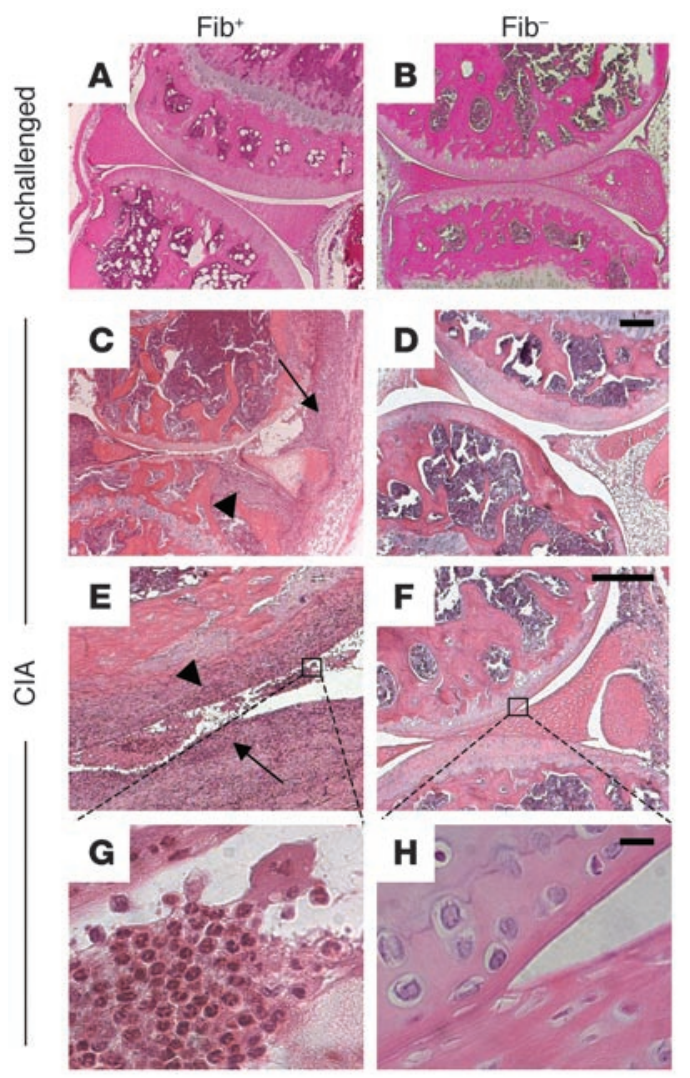

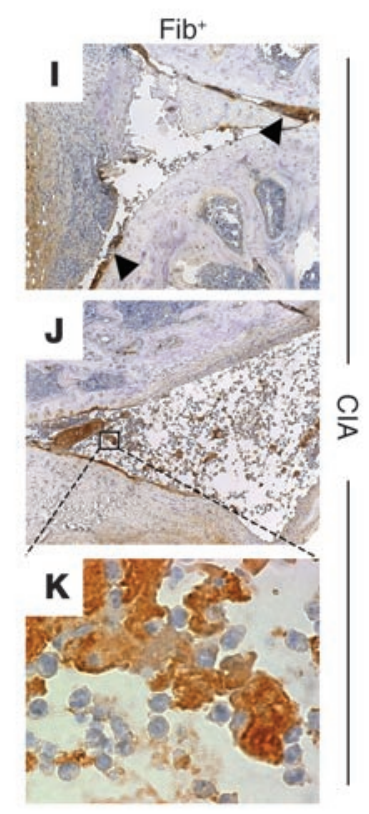

\section{Figure 3}

Diminished joint pathology within the knees of $\mathrm{Fib}^{-}$ mice immunized with CII. (A-H) Representative hematoxylin and eosin-stained knee joint sections prepared from unchallenged ( $\mathbf{A}$ and $\mathbf{B}$ ) and $\mathrm{Cll}$-immunized (C-H) $\mathrm{Fib}^{+}$and Fib- mice. At 40 days after initial challenge, the knee joints collected from $\mathrm{Fib}^{+}$mice exhibited extensive destruction of the joint, characterized by widespread synovial hyperplasia (C, arrow), and considerable erosion, if not obliteration, of cartilage on articular surfaces (C and $\mathbf{E}$, arrowheads) and within the meniscus. Neutrophil-rich inflammatory exudates were frequently observed within the synovial fluid of knees collected from $\mathrm{Fib}^{+}$mice with $\mathrm{CIA}(\mathbf{E}$ and $\mathbf{G})$. In contrast, knee joints collected in parallel from Cllimmunized $\mathrm{Fib}^{-}$mice typically displayed more normal architecture with intact and smooth articular surfaces (D, F, and H). (I-K) Immunohistochemical detection of fibrin(ogen) within knee sections prepared from $\mathrm{Fib}^{+}$ mice (brown stain). Note the strong fibrin(ogen) deposition on eroding articular surfaces (I, arrowheads) and as a component of neutrophil-rich rice bodies within the joint space ( $\mathbf{J}$ and $\mathbf{K})$. Scale bar: $200 \mu \mathrm{m}(\mathbf{A}-\mathbf{F}, \mathbf{I}$, and $\mathbf{J}) ; 10 \mu \mathrm{m}(\mathbf{G}, \mathbf{H}$, and $\mathbf{K})$. microscopic features of disease (data not shown). Fibrin staining was predictably missing from all joints collected from $\mathrm{Fib}^{-}$mice, confirming the specificity of the staining protocol (Figure $2 \mathrm{~L}$ ).

To determine whether the amelioration of arthritis observed in the distal joints of $\mathrm{Fib}^{-}$mice was evident within other joints, knee joints were harvested from cohorts of $\mathrm{Fib}^{+}$and $\mathrm{Fib}^{-}$mice for microscopic evaluation 40 days after the primary CII immunization. Representative knee joint sections are shown in Figure 3, and a more extensive gallery of knee joint views is shown in Supplemental Figure 2. Knee joints from unchallenged $\mathrm{Fib}^{+}$and $\mathrm{Fib}^{-}$mice appeared microscopically normal with intact joint architecture (Figure 3, A and B), further establishing that fibrinogen deficiency alone has no effect on joint development. However, $\mathrm{Fib}^{+}$mice with CIA displayed profound pathological features, including pronounced inflammatory cell infiltrates within the synovial tissue, neutrophil-rich aggregates within the joint space, massive synovial hyperplasia, and expanded joint space caused by edema (Figure 3, C, E, and G, and Supplemental Figure 2). The cartilage of articular surfaces was substantially degraded in most knee joints from $\mathrm{Fib}^{+}$mice (Figure 3E). Significant bone erosion by an invading pannus composed of newly formed granulation tissue was observed in the knee joints of $\mathrm{Fib}^{+}$mice, often penetrating to the growth plate and into the marrow (Supplemental Figure 2, J, N, and R). Fibrin deposition was once again a conspicuous feature of the arthritic knee joints of CII-immunized $\mathrm{Fib}^{+}$mice. Intense fibrin staining was observed along eroded articular surfaces, within hyperplastic inflammatory cell-rich synovium, and as a component of insoluble particulate rice bodies within the joint space of affected $\mathrm{Fib}^{+}$mice (Figure 3, I-K). The colocalization of fibrin with leukocytes observed in the $\mathrm{Fib}^{+}$mice was easily recognized within the joint space of CIA-challenged mice, in which morphologically distinct neutrophils were found intermingled with fibrin-rich matrices (Figure 3, E, G, J, and K). In contrast, qualitative microscopic analyses of knee joints collected from $\mathrm{Fib}^{-}$mice revealed far less evidence of arthritic disease, and some joints appeared largely normal. Those affected knees in $\mathrm{Fib}^{-}$mice typically displayed only mild synovial hyperplasia and inflammation (Figure 3D and Supplemental Figure 2, L and P). Rarely was significant cartilage degradation or bone erosion observed in knee joints of Fib- mice (Figure 3, D, F, and $\mathrm{H}$, and Supplemental Figure 2).

A quantitative comparison of knee joint histopathology was performed using knee joint sections collected from cohorts of $\mathrm{Fib}^{+}$and $\mathrm{Fib}^{-}$mice 40 days after primary $\mathrm{CII}$ immunization. Three independent investigators unaware of mouse genotype evaluated knee joint sections with regard to the following pathological features: inflammation, synovial hyperplasia, edema, pannus formation, and cartilage/bone destruction (see Methods). Consistent with qualitative analyses, the composite histopathology score of Fib $^{-}$mice showed a statistically significant diminution relative to that for $\mathrm{Fib}^{+}$mice (Figure 4A). Furthermore, every individual disease parameter scored was significantly diminished in $\mathrm{Fib}^{-}$mice (Figure 4B), consistent with the view that the genetic elimination of fibrin(ogen) broadly limits the pathological process.

Morphometric analyses of trichrome-stained knee joint sections (Figure 5A) were performed to quantitatively compare the fraction of cross-sectional articular surfaces remaining intact in cohorts of CIA-challenged $\mathrm{Fib}^{+}$and $\mathrm{Fib}^{-}$mice at day 40. Fib mice exhibited significantly less articular surface cartilage erosion than did the $\mathrm{Fib}^{+}$cohorts (Figure 5B). Thus, as observed for distal paw joints, the knee joints of CII-immunized $\mathrm{Fib}^{+}$mice showed far more advanced CIA relative to $\mathrm{Fib}^{-}$mice. These data suggest that 


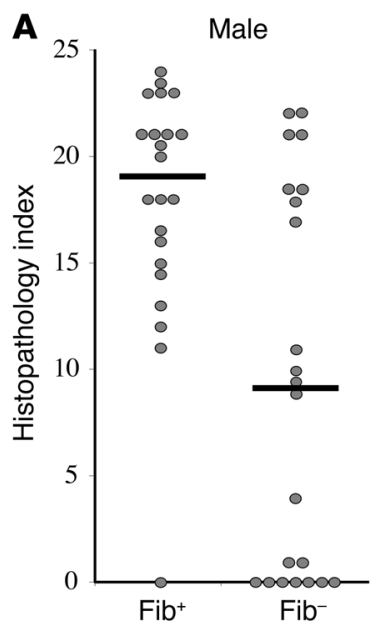

B

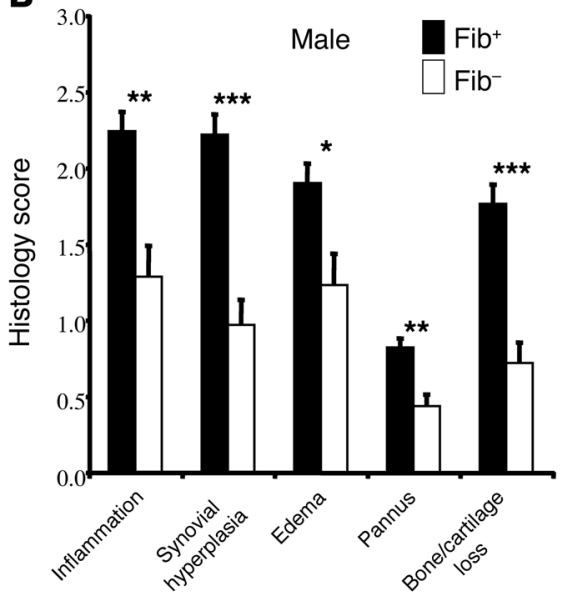

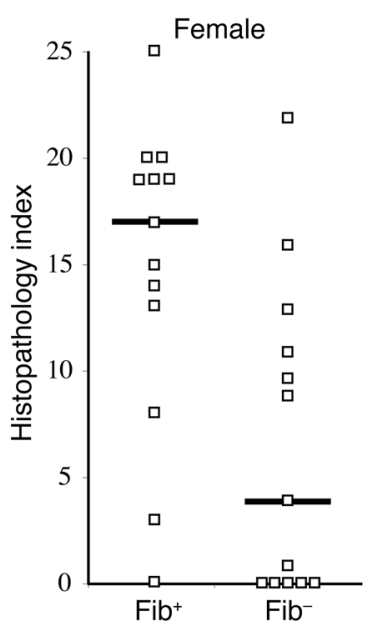

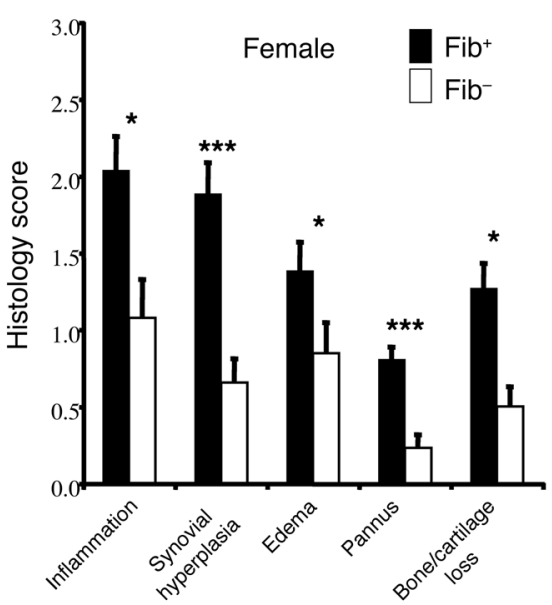

\section{Figure 4}

Fibrinogen deficiency results in diminished microscopic evidence of disease within knee joints of CIA-challenged mice. Knee joint sections from individual groups of $\mathrm{Fib}^{+}$and Fib- mice that were either all male $(n=22$ mice, 44 knees per genotype) or all female ( $n=13$ mice, 26 knees per genotype) mice were processed for histological evaluation on day 40 of the CIA protocol. (A) Composite histopathology index (the sum of scores for individual disease parameters) for each $\mathrm{Fib}^{-}$and $\mathrm{Fib}^{+}$mouse immunized with $\mathrm{Cll}$. Symbols denote values for individual mice; bars denote median values. $P<0.002$, $P<0.005$ between groups in male and female mice, respectively; Mann-Whitney $U$ test. (B) Scores for each of the knee histology evaluation parameters. Every disease parameter evaluated was significantly reduced in $\mathrm{Fib}^{-}$ relative to $\mathrm{Fib}^{+} \mathrm{Cll}$-immunized mice. Data are mean \pm SEM. ${ }^{*} P<0.05,{ }^{* *} P<0.0005$, ${ }^{* * *} P<0.0001$, Student's $t$ test. fibrin(ogen) either plays an important role in early events leading to inflammatory joint disease or is an essential modifier of multiple pathological processes in arthritis.

Fibrin (ogen) is not required for the adaptive immune response to CII or for leukocyte trafficking to arthritic joints. To begin to establish the mechanism by which fibrin(ogen) influences the progression of CIA, we first evaluated whether fibrin(ogen) is a general determinant of the adaptive immune response following CII immunization. Plasma was collected from $\mathrm{Fib}^{+}$and $\mathrm{Fib}^{-}$mice on day 40 following primary $\mathrm{CII}$ immunization and analyzed for anti-CII antibody titers. As shown in Figure 6A, anti-CII antibody titers of total IgG and the $\mathrm{IgG}_{1}$ and $\mathrm{IgG}_{2 \mathrm{~A}}$ subclasses were found to be similar between $\mathrm{Fib}^{+}$ and $\mathrm{Fib}^{-}$mice. The development of cellular immunity was also not impaired in $\mathrm{Fib}^{-}$mice. Splenocytes prepared from mice 35 days following primary $\mathrm{CII}$ immunization were analyzed for their ability to respond and proliferate in response to CII. As shown in Figure 6B, spleen cells from $\mathrm{Fib}^{+}$and $\mathrm{Fib}^{-}$mice responded similarly to $\mathrm{CII}$ exposure. Consistent with our expectations, $T$ cells prepared from both $\mathrm{Fib}^{-}$and $\mathrm{Fib}^{+}$mice exhibited little cell proliferation in the absence of any stimulus and showed an equally robust proliferative response when directly stimulated with an activating $\mathrm{T}$ cell receptor antibody (Figure 6B). Similar proliferation results were obtained with lymphocyte preparations derived from popliteal and inguinal lymph nodes collected from $\mathrm{Fib}^{-}$and $\mathrm{Fib}^{+}$mice (data not shown). Together these data indicate that $\mathrm{Fib}^{-}$mice are able to mount an adaptive immune response similar to that of control mice in the context of CII immunization.

Fibrin(ogen) has been shown not to be essential for leukocyte trafficking in contexts such as thioglycolate-induced peritonitis (Supplemental Figure 3 and ref. 14). Microscopic inspection of both knee and distal joints from CIA-challenged Fib $^{-}$mice also established that fibrin(ogen) was not strictly required for leukocyte trafficking into joints (Figures 2 and 3 and Supplemental Figure 2). To further examine the potential contribution of fibrin(ogen) to neutrophil trafficking and/or accumulation within joints at early and late stages of CIA, the levels of granulocyte-specific myeloperoxidase (MPO) activity in whole-joint extracts from unchallenged and CII-challenged $\mathrm{Fib}^{+}$and $\mathrm{Fib}^{-}$mice were assessed. Although indirect, MPO activity has been frequently shown to reflect total neutrophil levels within tissues, including murine arthritic joints (23). The MPO analysis within hind paw joints at day 28 after initial CII immunization (Figure 6C) indicated that neutrophil infiltrates were appreciably higher in challenged mice relative to unchallenged mice regardless of genotype. However, consistent with histopathologic observations (Supplemental Figure 2), fibrin(ogen) was not required for neutrophil trafficking early in disease, because MPO activity was similar in paws from mice of both genotypes harvested at day 28 of CIA. Nevertheless, consistent with the working hypothesis that fibrin supports local leukocyte activation events that can lead to secondary leukocyte recruitment, at day 40 after initial CII immunization MPO activity in the distal joints of 
A

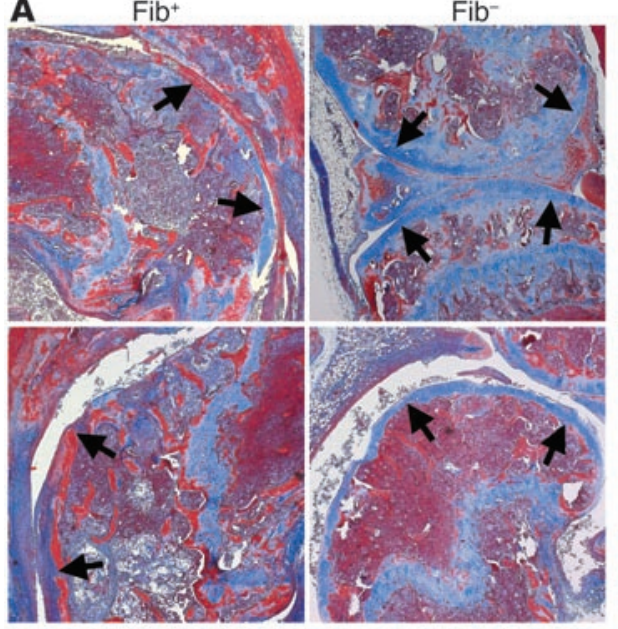

B

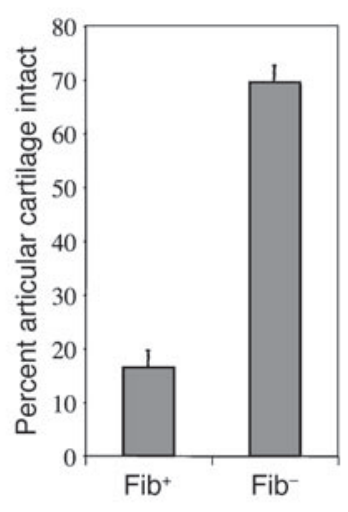

\section{Figure 5}

Diminished knee joint cartilage degradation in $\mathrm{Fib}^{-}$mice with $\mathrm{ClA}$ relative to $\mathrm{Fib}^{+}$mice with $\mathrm{CIA}$. (A) Representative knee joint sections prepared from $\mathrm{Fib}^{+}$and $\mathrm{Fib}^{-}$mice with $\mathrm{CIA}$ on day 40 of the protocol and stained with Masson's trichrome. Note the absence of contiguous Alcian blue stain, and thus the absence of cartilage, along much of the articular surface of $\mathrm{Fib}^{+}$mice, whereas the majority of the articular surface of $\mathrm{Fib}^{-}$mice stained positive for Alcian blue. Arrows denote the position of the articular surface. (B) Percent of knee joint articular surface cartilage remaining intact and smooth on the tibias of $\mathrm{Fib}^{+}(n=12)$ and $\mathrm{Fib}^{-}(n=9)$ mice with $\mathrm{CIA}$. The articular surface length staining positive for Alcian blue was measured by morphometric analysis and compared with the total length of the articular surface of the tibia. Results are shown as mean \pm SEM percent of Alcian blue staining. $P<0.00001$, Student's $t$ test.
$\mathrm{Fib}^{-}$mice was significantly less than that of $\mathrm{Fib}^{+}$mouse cohorts challenged in parallel (Figure 6C). Similar results were obtained for knee joints harvested from $\mathrm{Fib}^{+}$and $\mathrm{Fib}^{-}$mice (data not shown).

Mice expressing a mutant form of fibrinogen lacking the platelet integrin receptor $\alpha_{I I b} \beta_{3}$ binding motif develop CIA similar to wild-type mice. One plausible mechanism by which fibrin(ogen) could contribute to inflammatory joint disease is by supporting platelet deposition and the release of platelet-associated inflammatory mediators within the vasculature serving challenged joints (24). To determine whether platelet receptor engagement of fibrinogen is coupled to inflammatory joint disease, we pursued comparative studies of CIA in wild-type control mice and mice with a knockin mutation in the endogenous fibrinogen $\gamma$ chain gene that results in a mutant form of fibrinogen lacking the platelet integrin receptor $\alpha_{\mathrm{II}} \beta_{3}$ binding motif $\left(\right.$ Fib $\left.\gamma^{\Delta 5}\right)(20)$. Unlike Fib ${ }^{-}$mice, Fib $\gamma^{\Delta 5}$ mice developed inflammatory joint disease with a rate, incidence, and severity similar to that of wild-type mice. The arthritic index (Figure 7A) and arthritic severity of paws (Figure 7B) for Fib $\gamma^{\Delta 5}$ mice were comparable to those of wild-type mice throughout the 42-day course of the analysis. Thus, while fibrin(ogen) is a determinant of inflammatory joint disease, the platelet integrin receptor binding feature of fibrinogen is not essential for the development and progression of CIA.

Mice expressing a mutant form of fibrinogen lacking the integrin $\alpha_{M} \beta_{2}$ binding motif have diminished CIA. Fibrin and immobilized fibrinogen are established ligands for the leukocyte $\beta_{2}$ integrin receptor $\alpha_{M} \beta_{2}$ (Mac-1, CD11b/CD18) and are known to modify leukocyte function in vitro through an $\alpha_{M} \beta_{2}$-dependent mechanism. Thus, one attractive hypothesis is that local fibrin deposition is mechanistically coupled to joint inflammation and/or degeneration by promoting $\alpha_{M} \beta_{2}$-mediated leukocyte activation events. We previously generated a mouse line carrying a knockin mutation in the endogenous fibrinogen $\gamma$ chain gene that selectively eliminated the fibrinogen $\alpha_{M} \beta_{2}$ binding motif while preserving normal clotting function, platelet integrin engagement, and thrombus formation (Fib $\gamma^{390-396 \mathrm{~A}}$ ) (14). Comparative studies of CIA in Fib $\gamma^{390-396 \mathrm{~A}}$ and wild-type mice revealed that the selective elimination of the $\alpha_{M} \beta_{2}$ binding element on fibrin(ogen) was nearly as beneficial in reducing joint disease as the complete elimination of fibrin(ogen), despite the fact that there is neither any impediment in coagulation nor any apparent hemorrhagic phenotype or risk in Fib $\gamma^{390-396 \mathrm{~A}}$ mice. Fib $\gamma^{390-396 \mathrm{~A}}$ cohorts exhibited significantly diminished arthritic index (Figure 8A) and severity (Figure $8 \mathrm{~B}$ ) in the distal joints over the entire observation period (days 28-42). Consistent with the overt macroscopic findings, a detailed microscopic analysis of knee joints at day 42 revealed that Fib $\gamma^{390-396 A}$ mice exhibited significantly less joint pathology (e.g., inflammation, synovial hyperplasia, edema, pannus, and bone/cartilage loss) than that observed in knee sections from wild-type mice (Figure 8, C and D). Notably, plasma fibrinogen levels in Fiby $\gamma^{390-396 \mathrm{~A}}$ mice were comparable to those of wild-type mice in both unchallenged (14) and CIA-challenged mice (Supplemental Figure 4), as shown by immunological analyses. However, in contrast to that in CIA-challenged Fib ${ }^{-}$mice, fibrin deposition within affected joints of challenged Fib $\gamma^{390-396 \mathrm{~A}}$ mice was readily detected (Figure 8C). Nevertheless, as with the other macroscopic and microscopic disease parameters, CIA-challenged Fib $\gamma^{390-396 \mathrm{~A}}$ mice had significantly reduced cartilage erosion relative to challenged wild-type mice, as determined by morphometric analysis of trichrome-stained joint sections taken at day 42 of CIA (Figure 8, E and F). Taken together, these results suggest that a key functional property of fibrin integral to proarthritic activity in CIA is the capacity of this provisional matrix to engage the leukocyte integrin receptor $\alpha_{M} \beta_{2}$.

Fib ${ }^{-}$and Fiby ${ }^{390-396 A}$ mice with CIA exhibitreduced local levels of proinflammatory cytokines. The general amelioration of CIA in $\mathrm{Fib}^{-}$and Fiby ${ }^{390-396 \mathrm{~A}}$ mice retaining appreciable leukocyte trafficking (Figures 2, 3, and 6 and Supplemental Figure 3) suggests a mechanism whereby $\alpha_{M} \beta_{2^{-}}$ mediated leukocyte engagement of fibrin within joints supports inflammatory cell activation events associated with target recognition. Under this general model, the local expression of inflammatory mediators within the joints of CIA-challenged $\mathrm{Fib}^{-}$and Fib $\gamma^{390-396 \mathrm{~A}}$ mice would be expected to be reduced relative to that of control mice. Consistent with this notion, cytokine mRNA levels in joints at day 28 of CIA were diminished by 1.7-, 9-, and 26-fold for TNF- $\alpha$, IL-1 $\beta$, and IL-6, respectively, in challenged $\mathrm{Fib}^{-}$mice relative to challenged $\mathrm{Fib}^{+}$mice, and each of these differences was statistically significant (Figure 9, A-C). Similar results were obtained for Fib $\gamma^{390-396 \mathrm{~A}}$ mice relative to wild-type mice: cytokine levels were diminished 5.4-, 15-, and 25-fold for TNF- $\alpha$, IL-1 $\beta$, and IL-6, respectively (Figure 9, D-F). Furthermore, at day 42 , IL-1 $\beta$ and IL- 6 were diminished by a statistically significant 6.1- and 48-fold, respectively, for CIA-challenged Fib $\gamma^{390-396 \mathrm{~A}}$ mice relative to wild-type mice (Figure 9, E and F). There was no genotype-dependent difference in TNF- $\alpha$ levels at day 42. It should be noted that individual paws collected from control 
A

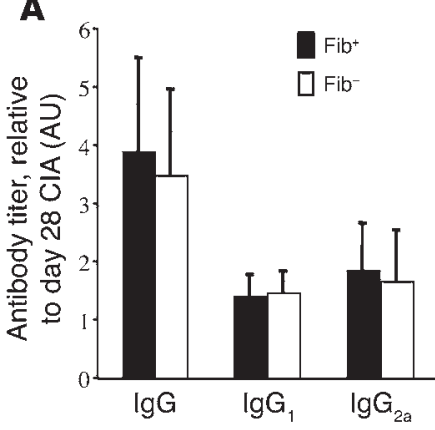

B

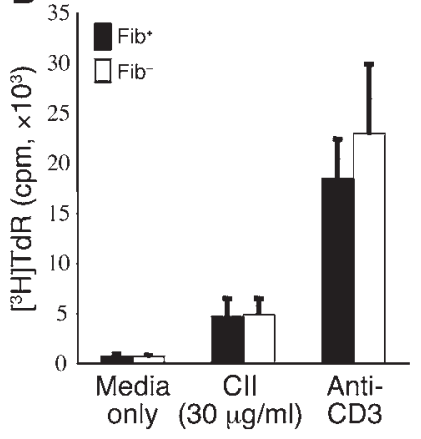

C

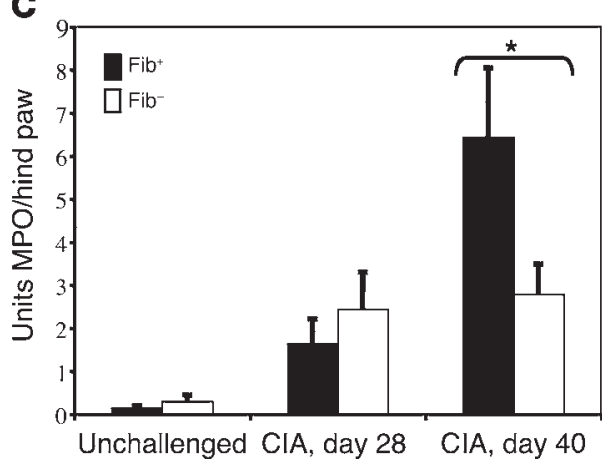

and Fib $\gamma^{390-396 A}$ mice that shared a similar clinical arthritic index also had similar cytokine mRNA levels (data not shown), consistent with the view that fibrinogen is important, but not essential, to mount a robust inflammatory response within joints.

$T N F-\alpha-$-induced inflammatory joint disease is not dependent on fibrin (ogen). Given that the absence of the fibrinogen $\alpha_{M} \beta_{2}$ binding motif resulted in decreased overall local production of inflammatory mediators, one attractive hypothesis is that fibrin(ogen) acts, at least in part, upstream of these proinflammatory cytokines, perhaps serving as a local cue for stimulating the production and/or release of these agents in the joint space. TNF- $\alpha$ is of particular interest in this regard because (a) this cytokine has a preeminent role in human disease and is elevated early in the progression of inflammatory joint disease $(1,25)$; (b) it directly controls inflammatory cell function and regulates the release of many other cytokines $(1,5)$; (c) it stimulates synovial cell proliferation (26); and (d) overexpression is sufficient to initiate severe, early-onset arthritis in mice (21). $\operatorname{Tg} 197$ mice expressing exuberant human TNF- $\alpha$ develop spontaneous, and ultimately debilitating, polyarthritis with $100 \%$ penetrance $(6,21)$. To test the hypotheses that the importance of fibrin(ogen) in inflammatory joint disease might be context dependent and superseded in mice with constitutive TNF- $\alpha$ expression, we examined the effect of fibrinogen deficiency on inflammatory joint disease in $\operatorname{Tg} 197$ mice. Fib- mice were $^{-}$ crossed with transgenic animals to generate cohorts of TNF- $\alpha$ transgene-positive mice with and without fibrinogen. As shown in Figure 10, $A$ and $B$, the arthritic index was not significantly different in either 8- or 10- week-old mice, a time frame in which arthritic disease becomes severe and even debilitating. Unlike CIA, fibrinogen deficiency, if anything, resulted in slightly worse

\section{Figure 7}

\section{Figure 6}

Fibrinogen deficiency does not alter anti-CII antibody production, T cell reactivity, or neutrophil accumulation in joints of mice with $\mathrm{CIA}$. (A) Determination of anti-CII specific IgG antibody titers by ELISA using plasma harvested at day 40 of the $\mathrm{ClA}$ protocol from $\mathrm{Fib}^{+}$and $\mathrm{Fib}^{-}$mice ( $n=12$ per group). Titers are expressed as arbitrary units relative to titers found in wild-type DBA/ 1 mice at day 28 of CIA. Results are expressed as mean \pm SEM. (B) Cellular response to CII. Spleen cells were harvested from $\mathrm{Fib}^{+}$and $\mathrm{Fib}^{-}$mice ( $n=3$ per group) at day 40 of the $\mathrm{ClA}$ protocol and stimulated with $30 \mu \mathrm{g} / \mathrm{ml} \mathrm{Cll}$ or anti-CD3 activating T cell receptor antibody. Results are mean $\pm \mathrm{SEM}$ of $\left[{ }^{3} \mathrm{H}\right] \mathrm{TdR}$ incorporated in cpm. (C) Determination of neutrophil-derived MPO activity in total protein extracts from hind paws of $\mathrm{Fib}^{+}$and $\mathrm{Fib}^{-}$mice that were unchallenged ( $n=4$ per genotype), at day 28 of CIA ( $n=8$ per genotype), and at day 40 of $\mathrm{CIA}(n=16$ per genotype). Results are mean \pm SEM. ${ }^{\star} P<0.03$, Student's $t$ test.

TNF- $\alpha$-driven arthritic disease, perhaps because of alterations in vascular integrity in $\mathrm{Fib}^{-}$mice with inflammatory joint damage. Consistent with overt macroscopic evaluations, histological examination of knee joint sections taken from 10-week-old TNF- $\alpha$ transgene-positive mice with and without fibrinogen revealed a similar pattern of severe joint pathology. Inflammatory cell infiltrates, massive synovial hyperplasia, cartilage and bone loss, and in many cases complete loss of joint architecture were observed in mice of both genotypes (Figure 10C). Quantitative analysis of knee joint histopathology confirmed that disease progression was similar in transgene-positive $\mathrm{Fib}^{-}$and $\mathrm{Fib}^{+}$mice (Figure 10D). Furthermore, the histology scores for each feature of disease were comparable between genotypes, suggesting that fibrin(ogen) did not differentially affect the progression of any single aspect of TNF- $\alpha$-driven disease (Figure 10E). Given that fibrin(ogen) is a potent proarthritic factor in the context of CIA, these results imply that fibrin(ogen) controls the progression of inflammatory joint disease through a regulatory pathway lying upstream of local TNF- $\alpha$ production and/or that exuberant TNF- $\alpha$ can supersede local fibrin deposition in controlling inflammatory disease processes in the joint.

\section{Discussion}

These studies of arthritis in mice with selected alterations in fibrinogen have directly established that: (a) fibrin(ogen) is an important determinant of inflammatory joint disease, (b) fibrin(ogen) depo-
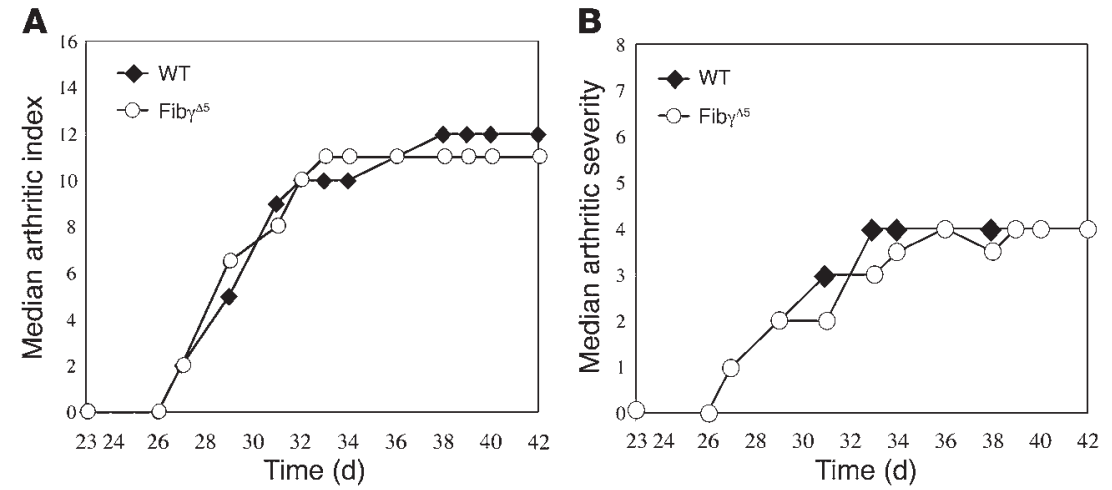

Fib $\gamma^{\Delta 5}$ mice develop similar CIA to control mice. Median arthritic index (A) and median arthritic severity (B) of paws from 2 combined experiments using cohorts of wild-type $(n=31)$ and Fib $\gamma^{\Delta 5}(n=30)$ mice. 
A

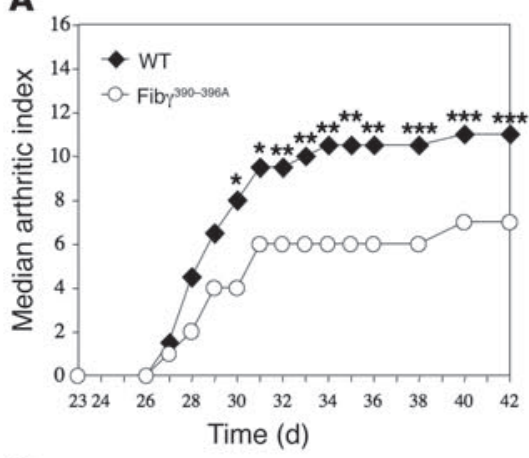

C

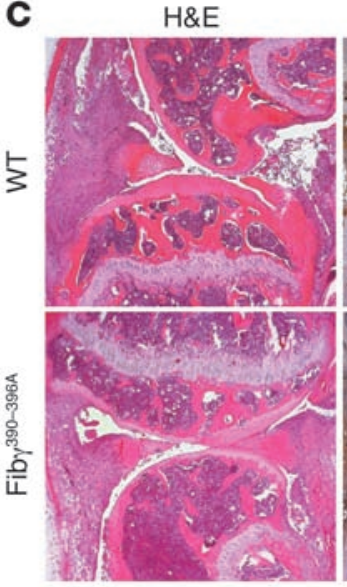

E

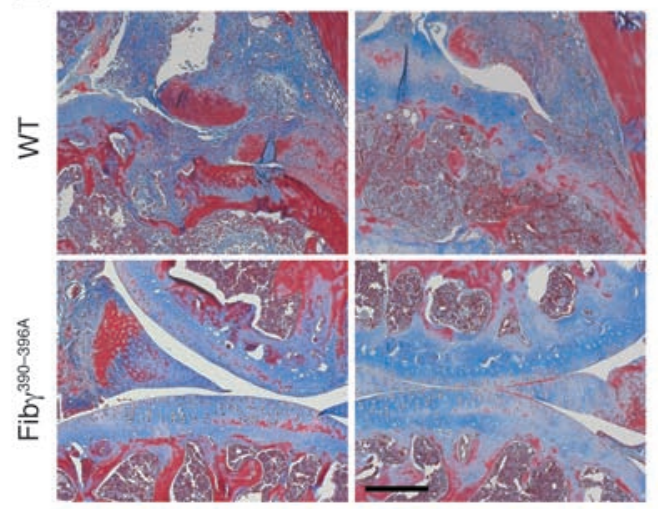

B

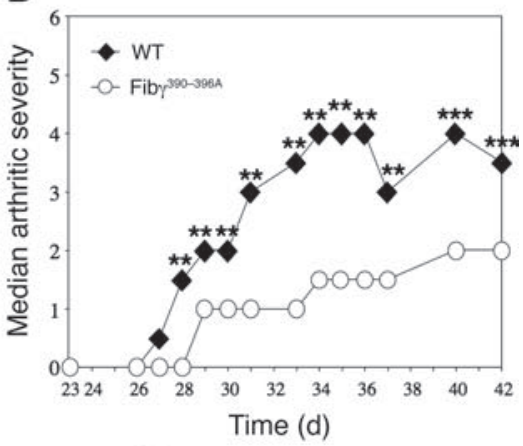

D

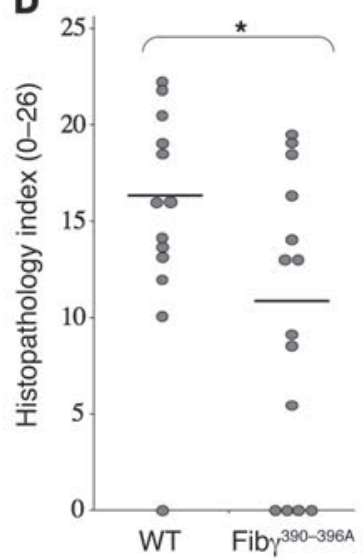

F

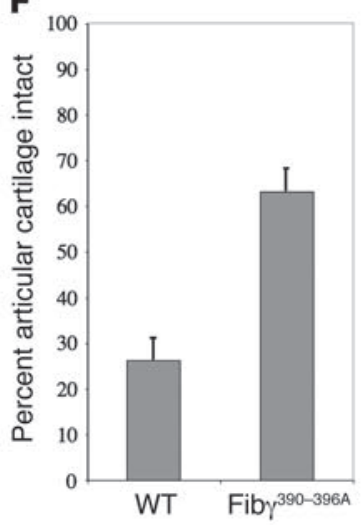

Figure 8

Diminution of CIA in Fib $\gamma^{390-396 A}$ knockin mice. (A) Median arthritic index of paws from a representative experiment in which a cohort of wild-type DBA/1 $(n=16)$ and Fib $\gamma^{390-396 \mathrm{~A}}(n=17)$ mice were immunized with CII. (B) Median arthritic severity of paws from 2 combined experiments using cohorts of wildtype DBA/1 and Fib $\gamma^{390-396 \mathrm{~A}}$ mice ( $n=32$ per group). ${ }^{\star} P<0.05,{ }^{* *} P<0.02$, ${ }^{* * *} P<0.005$, Mann-Whitney $U$ test. (C) Representative knee joint sections from wild-type and Fib ${ }^{390-396 A}$ mice stained either with hematoxylin and eosin or immunohistochemically for fibrin. Fib ${ }^{390-396 A}$ mice generally had reduced knee joint pathology relative to knee joints from wild-type mice, despite the fact that fibrin deposition remained a prominent feature of knee joints from Fib $\gamma^{390-396 A}$ mice. (D) Histopathology index analysis of knee joint sections from wild-type $(n=13)$ and Fib $\gamma^{390-396 A}$ $(n=14)$ mice. $P<0.05$, Mann-Whitney $U$ test. $(E)$ Representative knee joint sections from wild-type and Fiby ${ }^{390-396 \mathrm{~A}}$ mice at day 42 of $\mathrm{CIA}$ stained with Masson's trichrome. (F) Morphometric analysis of intact tibial surface cartilage of CIA day 42 wild-type and Fib ${ }^{390-396 A}$ mice. $P<0.00001$, Student's $t$ test. Scale bars: $200 \mu \mathrm{m}(\mathbf{C}) ; 100 \mu \mathrm{m}(\mathbf{E})$. sition within joints drives arthritic disease via mechanisms linked to the leukocyte integrin receptor $\alpha_{M} \beta_{2}$ binding motif, and (c) the impact of fibrin(ogen) on the progression of joint disease is context dependent. In the experimental setting of CIA, the genetic elimination of fibrin(ogen) significantly diminished the development of overt, clinically evident arthritis and ameliorated the entire spectrum of pathological features of disease when tissue sections were examined microscopically. However, fibrinogen deficiency did not diminish the adaptive immune responses (e.g., T cell response and anti-CII antibody production) and was compatible with leukocyte trafficking following CIA. Thus, fibrin-rich matrices appear to serve as powerful modifiers of the local inflammatory events leading to joint degeneration. While disease severity was much reduced within cohorts of $\mathrm{Fib}^{-}$mice relative to $\mathrm{Fib}^{+}$controls, individual $\mathrm{Fib}^{-}$mice that developed disease were encountered. Thus, neither fibrinogen nor fibrin is strictly required for the development of arthritis, and inflammatory pathways must still exist that can supersede the fibrinogen-dependent process(es) in arthritis (see below). Complementary CIA studies in mice that express mutant forms of fibrinogen lacking selected integrin receptor engagement motifs demonstrated that the elimination of the motif recognized by the leukocyte integrin receptor $\alpha_{M} \beta_{2}$ was sufficient to alleviate arthritis, despite the maintenance of full clotting. In contrast, the elimination of the platelet receptor $\alpha_{\mathrm{IIb}} \beta_{3}$ binding motif from fibrinogen had no apparent impact on the progression of inflammatory joint disease. The attenuation of collagen-induced arthritis in $\mathrm{Fib}^{-}$and Fib $\gamma^{390-396 \mathrm{~A}}$ mice was also associated with a significant diminution in the expression of key cytokines known to be important in the development of disease (e.g., TNF- $\alpha$, IL-1 $\beta$, and IL-6). An attractive working hypothesis that has emerged is that fibrin contributes to degenerative inflammatory changes within the joint by supporting $\alpha_{M} \beta_{2}$-dependent leukocyte activation programs, including the secondary expression of proinflammatory 
A

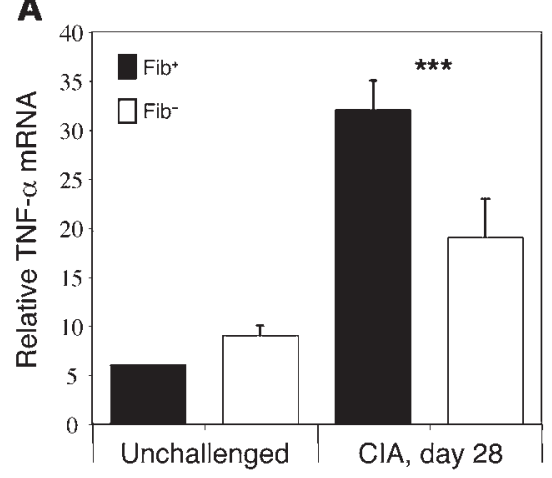

D

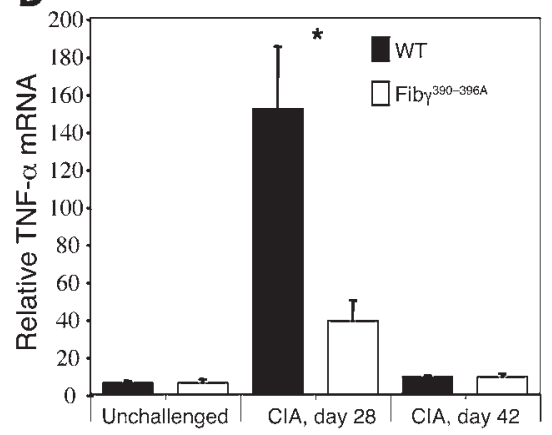

B

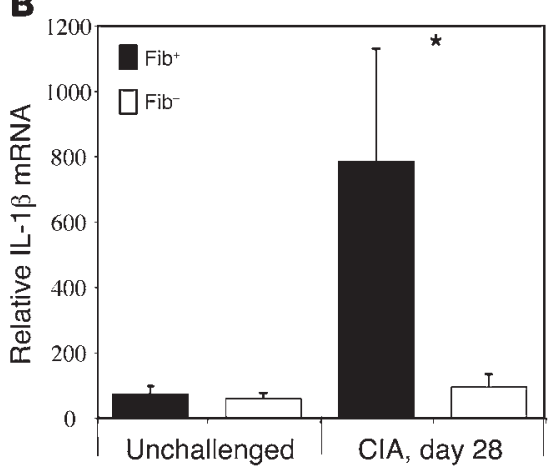

E

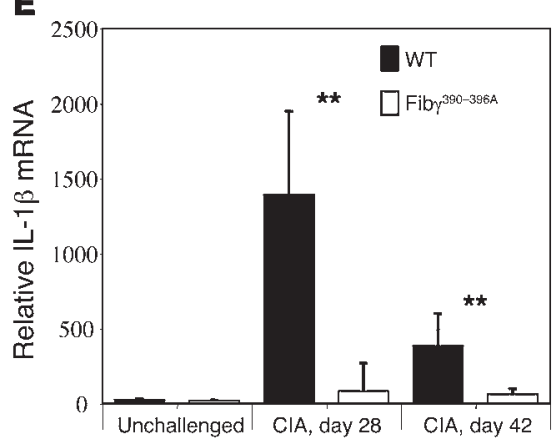

C

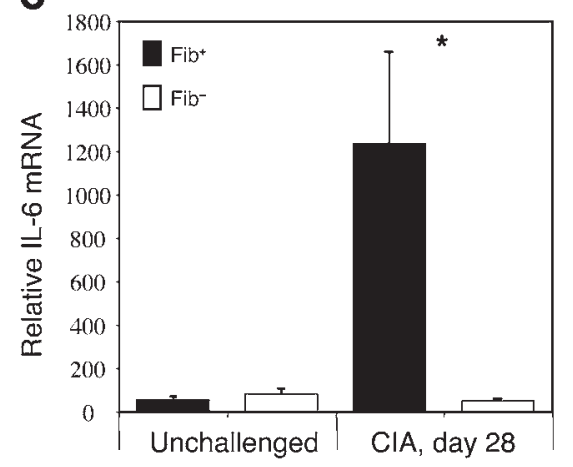

$\mathbf{F}$

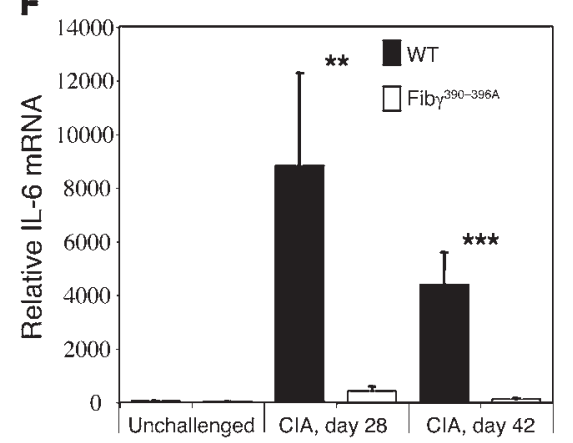

Figure 9

Paws from Fib- and Fiby ${ }^{390-396 A}$ mice have reduced message levels of proinflammatory cytokines in CIA. Quantitative PCR was used to determine the relative levels of TNF- $\alpha(\mathbf{A}$ and $\mathbf{D}), \mathrm{IL}-1 \beta(\mathbf{B}$ and $\mathbf{E})$, and IL-6 (C and F) from total RNA isolated from hind paws of mice. Paws from unchallenged $\mathrm{Fib}^{+}(n=8)$, Fib- $(n=8)$, wild-type $(n=4)$, and Fib $\gamma^{390-396 \mathrm{~A}}(n=4)$ mice were harvested as controls. Cll-immunized Fib ${ }^{+}$, Fib ${ }^{-}$, wild-type $(n=24)$, and CII-immunized Fiby ${ }^{390-396 A}(n=23)$ mice were analyzed at days 28 and 42 of the CIA protocol. Cytokine levels within each sample were normalized to GAPDH detected within that sample. Data are expressed as mean \pm SEM. ${ }^{\star} P<0.05,{ }^{\star \star} P<0.01,{ }^{* \star *} P<0.005$, Mann-Whitney $U$ test.

cytokines. Consistent with this view, arthritic disease was found to be fibrin(ogen) independent in the experimental context of mice expressing exuberant human TNF- $\alpha$.

A variety of mechanisms can be envisioned whereby fibrin(ogen) could influence the progression of inflammatory joint disease. First, as a key factor in the maintenance of vascular integrity and/or paten$c y$, fibrin thrombus formation within vessels serving joint tissues may further compromise already damaged arthritic joints by limiting blood flow and, ultimately, nutrient delivery and gas exchange. Second, the formation and deposition of antibodies to novel fibrin(ogen) derivatives (e.g., anti-citrullinated fibrinogen antibodies) might be a significant factor in joint disease (see below). Third, as a provisional matrix protein that supports tissue repair, the deposition and cellular organization of fibrin matrices within arthritic joints may drive inappropriate reparative processes. This could potentially lead to a vicious cycle of fibrin deposition, tissue reorganization, further loss of joint function, further mechanical and inflammatory damage, and further fibrin deposition. In this regard, fibrin and fibrin degradation products have been reported to have a remarkable array of biological activities that could contribute to inopportune reparative changes within damaged joints, including chemotactic, mitogenic, and angiogenic activities (27). Each of these mechanisms may be an important factor in human disease. However, the findings that Fiby $\gamma^{390-396 \mathrm{~A}}$ mice (a) carry levels of fibrinogen comparable to those of wild-type mice when both unchallenged and CIA challenged (present study and ref. 14), (b) retain full clotting function (14), and (c) exhibit a significant resistance to CIA similar to that of $\mathrm{Fib}^{-}$mice implies that any fibrin-dependent mechanisms linked to hemostasis may be of only modest importance in the context of standard CIA.

The genetic elimination of either fibrin(ogen) or $\alpha_{M} \beta_{2}$ has been shown to be compatible with leukocyte trafficking to multiple sites including the lung, skin, and peritoneal cavity in various experimental inflammatory contexts $(14,28-31)$. Here, we show that neutrophil migration into the joints of CIA-challenged mice is also not fundamentally compromised by the absence of fibrin(ogen). Rather, fibrinogen- $\alpha_{M} \beta_{2}$ interactions appear to regulate local leukocyte function. Multiple in vitro and in vivo studies have shown that fibrin(ogen)$\alpha_{M} \beta_{2}$ engagement can regulate the activities of macrophages, neutrophils, and other inflammatory cells, including cell adhesion, calcium mobilization, cytokine release, chemokine release, degranulation, oxidative response, phagocytosis, activation of ERK1/2, NFKBdependent transcription, and survival (10-13). For example, fibrinogen can trigger the full activation of neutrophils primed with TNF- $\alpha$ through an $\alpha_{M} \beta_{2}$-dependent mechanism resulting in degranulation, upregulation of cell surface adhesion molecules, increased phagocytosis, and delayed apoptosis (11). Neutrophils and macrophages expressing $\alpha_{M} \beta_{2}$ have been strongly implicated in the pathogenesis of arthritic disease based on depletion studies $(32,33)$. Therefore, an attractive working hypothesis is that fibrin(ogen) drives inflammatory joint disease by supporting local leukocyte "target recognition" and leukocyte activation events, presumably in concert with soluble immune mediators (e.g., cytokines and chemokines). However, it should be emphasized that multiple fibrin(ogen)-dependent processes may influence arthritic disease, and the importance of these 
A
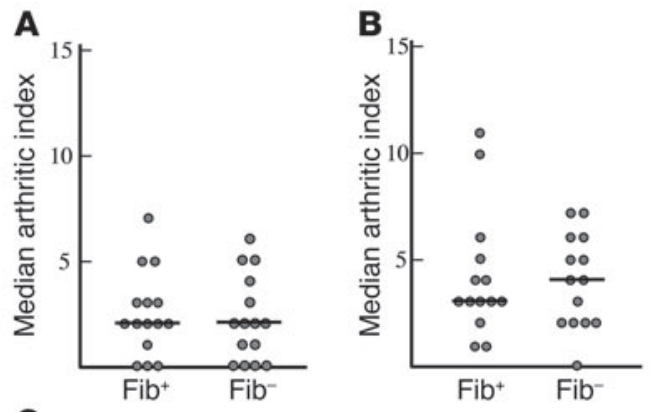

C
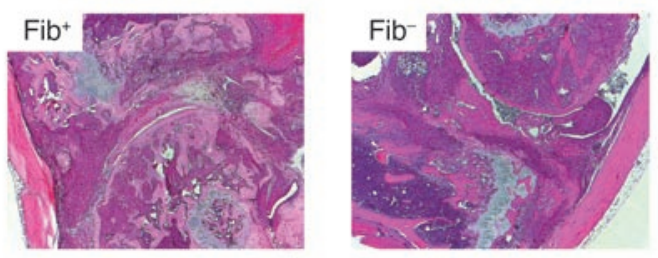

D

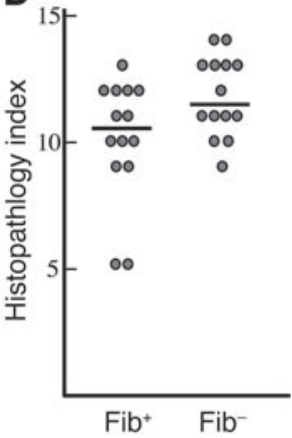

E

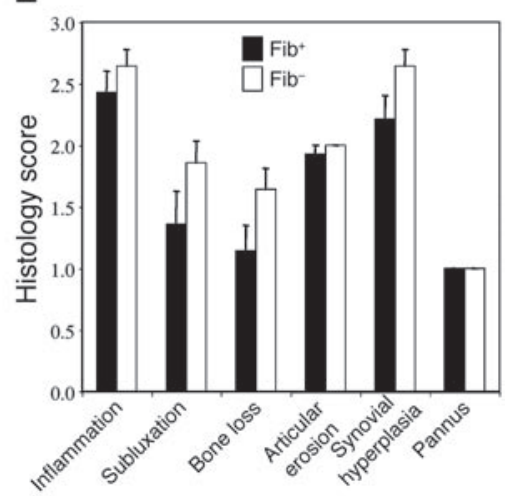

processes may vary from critical to modest based on joint location, mechanical stress, and underlying immunological derangements.

A concept that has recently gained increasing attention is that the conversion of arginine residues to citrulline by peptidylarginine deiminases on selected proteins is thought to generate novel epitopes that initiate antibody formation and subsequently result in immune complex-driven arthritis (34). Fibrinogen has been identified as a target of this modification, and the development of anti-citrullinated fibrinogen antibodies has been documented in both human RA patients and mice subjected to CIA (35-37). It was recently shown that direct administration of antibodies specific to citrullinated fibrinogen exacerbated disease initiated by an attenuated CIA protocol (38). Whatever the importance of anticitrullinated fibrin(ogen) antibodies as a determinant of arthritic disease, the present studies with Fib- mice establish that, at least in mice with CIA, the formation of anti-citrulline antibodies directed specifically at fibrin(ogen) is not required for the development of inflammatory joint disease. However, it is possible that there may be cooperation among multiple mechanisms in which fibrin(ogen) promotes the pathogenesis of arthritis. One attractive theory that couples previous findings on anti-citrullinated fibrin(ogen) antibodies and the present findings on fibrin(ogen)- $\alpha_{M} \beta_{2}$ interactions in arthritic disease is that local leukocyte activation events promote fibrin citrullination, secondary anti-citrullinated antibody formation, and, ultimately, immune complex-mediated arthritis, but examining this possibility will require additional analyses.

\section{Figure 10}

Fibrinogen deficiency has no effect on TNF- $\alpha$-driven inflammatory joint disease. (A and $\mathbf{B}$ ) Scatter plot of arthritic index scores of paws from 8-week-old (A) and 10-week-old (B) $\mathrm{Fib}^{+}$and $\mathrm{Fib}^{-}$mice overexpressing human TNF- $\alpha$. Bars indicate the median scores for each genotype. (C) Knee joint histology of representative sections taken from 10-weekold $\mathrm{Fib}^{+}$and $\mathrm{Fib}^{-}$mice overexpressing human TNF- $\alpha$ show identical joint destruction characterized by inflammatory cell infiltrate, robust synovial hyperplasia, articular cartilage erosion, and significant loss of bone. Quantitative evaluation of knee joint sections indicated a similar level of disease severity between TNF- $\alpha$-overexpressing $\mathrm{Fib}^{+}$and Fib- mice. (D) Scatter plot of composite histopathology index score of knee joint sections; bars indicate median values ( $\mathrm{Fib}^{+}, 10.5$; $\mathrm{Fib}^{-}, 11.5$; $P=0.09$, Mann-Whitney $U$ test). (E) Mean scores for histopathology criteria of knee joint sections showed no significant difference for any individual parameter.

Although the development of advanced and debilitating arthritic disease is a feature common to both TNF- $\alpha$-driven arthritis and CIA, these two experimental settings of joint disease have distinct etiologies, use unique mechanisms of pathogenesis, and, as shown here, have very distinct fibrin(ogen) dependencies. The contribution of proinflammatory cytokines to the pathogenesis of arthritis also is clearly distinct in CIA and Tg197 mice. For example, IL-1 $\beta$ has been shown to be critical for both CIA and TNF- $\alpha$-driven arthritis in mice (39). The genetic elimination of IL- 6 was reported to have no impact on the development of severe arthritic disease in $\operatorname{Tg} 197$ mice but results in near-complete protection from arthritis in the context of CIA (6). The distinct etiologies and cytokine-dependent pathways of disease in CIA and Tg197 mice, together with our finding that the local expression of cytokines (e.g., TNF- $\alpha$, IL-1 $\beta$, and IL-6) within challenged joints is sensitive to genetic alterations in fibrinogen, offer a plausible model consistent with all of our present findings and the working view that fibrin(ogen)-rich matrices can regulate inflammatory cell function. We propose that fibrinogen influences inflammatory joint disease at a control point upstream of the local expression of TNF- $\alpha$ and other important cytokines. Under this theory, the constitutive expression of a master immune modulator such as TNF- $\alpha$ would be expected to reduce or eliminate the disease dependence on fibrin(ogen), precisely what has been observed in Tg197 mice. The proposed placement of fibrin matrices upstream of the leukocyte activation events that support the secondary expression of fundamental soluble inflammatory mediators is also consistent with the profound diminution in arthritis observed in $\mathrm{Fib}^{-}$and Fib $\gamma^{390-396 \mathrm{~A}}$ mice in the context of CIA.

The finding that fibrin(ogen) is a powerful, but context dependent, contributor to inflammatory joint disease may provide a partial explanation for many seemingly conflicting reports on the role of fibrinolytic factors in inflammatory joint disease. Mice lacking either urokinase-type plasminogen activator or plasminogen were reported to develop greatly exacerbated AIA but markedly diminished CIA $(19,40-42)$. On the other hand, tissue-type plasminogen activator-deficient mice have been consistently resistant to arthritic disease challenge $(19,41)$. One potential variable that has been proposed to account for some of these seemingly incompatible findings is investigator-imposed joint trauma introduced in some settings (e.g., injection of antigen into the joint space in AIA), but not other settings (e.g., CIA) (19, 40-42). The present studies show that the importance of fibrinogen in arthritic disease can vary markedly in two distinct experimental contexts even when there is no investigator-imposed mechanical joint trauma. 
Similarly, plasmin-mediated proteolysis within joint spaces might be beneficial in some joint disease contexts by supporting prompt fibrin clearance, whereas excessive plasmin generation might aggravate disease in other contexts by participating in the direct or indirect proteolysis of extracellular matrix glycoproteins, collagen, or immune modulators (e.g., complement factors) $(40,42)$.

Fibrin deposition is carefully regulated both spatially and temporally at sites of injury, making it an ideal candidate to be a local, matrix-associated regulator of leukocyte target recognition leading to activation events in both physiological and pathological inflammatory contexts. The results of the present study directly establish that fibrin(ogen) is an important, albeit context-dependent, determinant of inflammatory joint disease. Together with our earlier finding that the elimination of the $\alpha_{M} \beta_{2}$ binding motif on fibrinogen impedes Staphylococcus aureus clearance in vivo (14), the present work shows that fibrin(ogen) is central to inflammatory processes in both physiological (e.g., antimicrobial host defense) and pathological (e.g., inflammatory joint disease) contexts. Finally, the available data suggest that fibrin(ogen) may be an attractive therapeutic target in the treatment of arthritis and other inflammatory diseases. This concept is also supported by previous studies showing that ancrod-mediated depletion of fibrinogen in mice can impede AIA (40). The findings presented here suggest that pharmacological interruption of fibrinogen- $\alpha_{M} \beta_{2}$ interactions might be efficacious in the treatment of arthritic disease. Because the feature of fibrinogen recognized by $\alpha_{M} \beta_{2}$ is functionally distinct from the features required for fibrin polymer formation, this could be accomplished without imposing any hemorrhagic risks.

\section{Methods}

Mice. Mice carrying a null mutation in the fibrinogen $A \alpha$ chain gene, resulting in complete Fib deficiency, and mice carrying selected knockin mutations in the fibrinogen $\gamma$ chain, resulting in the expression of either Fib $\gamma^{390-396 \mathrm{~A}}$ (lacking the leukocyte integrin receptor $\alpha_{M} \beta_{2}$ binding motif) or Fib $\gamma^{\Delta 5}$ (lacking the platelet integrin receptor $\alpha_{\mathrm{II}} \beta_{3}$ binding motif), were generated as previously described $(14,20,22)$. For CIA experiments, these lines were backcrossed 6 generations to DBA/1J (Jackson Laboratories) mice, and all-male or all-female mouse cohorts were enrolled in independent experiments and analyzed separately as indicated. In experiments with A $\alpha$ chain-null mice, control mice were heterozygous, because these animals (a) carry approximately $70 \%$ of the normal plasma fibrinogen level and (b) were found to be phenotypically identical to cohorts of homozygous wild-type DBA/ 1 mice in our CIA experiments (data not shown). For those studies involving Fib $\gamma^{390-396 \mathrm{~A}}$ or Fib $\gamma^{\Delta 5}$ mutations, homozygous mutant mice were paired with wild-type mice generated from within the same colony. $\operatorname{Tg} 197$ mice of the strain FVB/N were crossed with $\mathrm{C} 57 \mathrm{BL} / 6$ inbred $\mathrm{Fib}^{-}$mice. The resulting F1 progeny were intercrossed to produce $\mathrm{Fib}^{+}$and Fib- TNF- $\alpha$ transgene-positive mice. $\mathrm{Fib}^{+} \mathrm{Tg} 197^{+}$and $\mathrm{Fib}^{-} \mathrm{Tg} 197^{+}$littermate pairs were collected for evaluation. Mice were housed in the animal care facility at Cincinnati Children's Hospital Research Foundation, and the Cincinnati Children's Hospital Animal Care and Use Committee approved all experiments performed.

Collagen immunization. Male mice were injected intradermally with $100 \mu \mathrm{g}$ bovine CII (Elastin Products Co. Inc.) in CFA on days 1 and 21 of the protocol. Mice were evaluated for arthritis using an arthritic index macroscopic scoring system ranging from 0 to 4 ( 0 , no detectable arthritis; 1 , swelling and/or redness of paw or 1 digit; 2, 2 joints involved; 3, 3 joints involved; 4, severe arthritis of the entire paw and digits) (43). Mice were also evaluated based on the degree of paw swelling using an arthritic severity score (range, 0-2). Mice were sacrificed at specified time points following primary collagen immunization for joint histological, mRNA, or enzyme analysis (see below).
TNF- $\alpha$-driven inflammatory joint disease. Littermates of $\operatorname{Tg} 197$ mice that were also either $\mathrm{Fib}^{+}$or $\mathrm{Fib}^{-}$were macroscopically scored at 8 or 10 weeks of age for the number of affected digits in each paw. The total number of affected digits per animal was taken as the arthritic index. At these time points mice were also sacrificed, and knee joints were processed for histological evaluation (see below).

Histological analysis. Mouse tissues were fixed for 48 hours in $10 \%$ Neutral Buffered Formalin (Sigma-Aldrich) and then decalcified in TBD-2 (ThermoShandon) for 10 days. Sections ( $4 \mu \mathrm{m}$ ) of paraffin-embedded tissue were placed on Colorfrost Plus slides (Fisher Scientific). Tissue analyses focused on (a) horizontal sections (i.e., prepared in the plane of the fore paw) of the metacarpophalangeal joints, digits 3 and 4 , and (b) sagittal knee joint sections panning lateral to medial. Sections were stained with either hematoxylin and eosin or Masson's trichrome for evaluation. A semiquantitative histopathology analysis was performed for each knee joint based on the following scoring criteria for CIA: inflammation (range, 0-3), synovial hyperplasia (range, 0-3), edema (range, 0-3), pannus (range, 0-1), and bone/cartilage loss (range, 0-3). Scores were summed to determine the total histopathology index (range, 0-26) for knees of a given animal. For Tg197 transgenic mice, the following scoring criteria were used: inflammation (range, 0-3), subluxation (range, $0-3$ ), bone loss (range, $0-3$ ), articular erosion (range, $0-2$ ), synovial hyperplasia (range, $0-3$ ), and pannus (range, $0-1$ ). Representative sections were also immunostained for fibrin deposition using a rabbit anti-mouse fibrin polyclonal serum (44) and peroxidase/DAB development system.

Analysis of percent intact articular surface cartilage in knee joint sections was performed using morphometric image analysis software (Axiovision Release 4.5; Carl Zeiss Microimaging Inc.). Here, sagittal knee joint sections were stained with Masson's trichrome prior to image collection using an Axioplan 2 microscope (Carl Zeiss Microimaging Inc.). The total length of the articular surface of the tibia was measured from one end of the growth plate to the other and compared with the length of intact articular cartilage as indicated by smooth and uninterrupted Alcian blue staining.

Measurements of anti-CII antibody titers and in vitro T cell proliferation. Anti-CII antibody titers in plasma samples were determined by ELISA, as described previously (43). For proliferation assays, spleen cells $\left(5 \times 10^{5} /\right.$ well $)$ were plated in triplicate in DMEM containing $0.5 \%$ normal DBA/ 1 mouse serum at $37^{\circ} \mathrm{C}$ in 96 -well flat-bottomed microtiter plates that had been precoated with heat-denatured $\left(56^{\circ} \mathrm{C}\right.$ for $\left.10 \mathrm{~min}\right) \mathrm{CII}$ or anti-CD3-containing (antibody 2C11) cell culture supernatant as a positive control. Cells were incubated for $72 \mathrm{~h}$, followed by addition of $1 \mu \mathrm{Ci}$ of $\left[{ }^{3} \mathrm{H}\right]$ thymidine for an additional $18 \mathrm{~h}$, and then harvested. Incorporation of $\left[{ }^{3} \mathrm{H}\right]$ thymidine was measured per well and averaged for each triplicate. Background counts from unstimulated cells were subtracted from each group.

Determination of MPO activity in joint tissue extracts. Hind paws were snapfrozen in liquid nitrogen before being homogenized in buffer containing $0.5 \mathrm{M}$ sodium acetate, $\mathrm{pH}$ 6.0; $1 \%$ hexadecyl-trimethyl ammonium bromide; $20 \mathrm{mM}$ EDTA; and 1\% Nonidet P40 (all from Sigma-Aldrich). Lysates were cleared by centrifugation and assayed for MPO activity using $\mathrm{H}_{2} \mathrm{O}_{2}$ as a substrate and 3,3',5,5'-teramethylbenzidine as a chromogenic substrate. MPO activity was expressed in units based on dilutions of a known standard preparation of purified MPO from human leukocytes (Sigma-Aldrich).

$R N A$ isolation and real-time $m R N A$ quantification. Frozen paws $\left(-70^{\circ} \mathrm{C}\right.$; with skin intact) were homogenized with a polytron Tissue-Tearor (Biospec Products Inc.) in appropriate volumes of TRIzoL (Invitrogen), and RNA was extracted. RNAs were DNAse treated and further purified using the RNeasy MicroKit (Qiagen). cDNA was synthesized from $1 \mu \mathrm{g}$ of RNA using the SuperScript III First Strand Synthesis System for RT-PCR (Invitrogen). Real-time PCR was performed on serial dilutions of samples in 96-well plates on the iCycler iQ system (Bio-Rad). The 20- $\mu$ l reaction consisted of $500 \mathrm{nM}$ of primer, $4 \mu \mathrm{l}$ of cDNA, and $10 \mu \mathrm{l}$ of $2 \mathrm{X}$ SYBR Green Supermix (Bio-Rad), with iTaq 
DNA polymerase. Fluorescein dye was included for dynamic well factor collection on the iCycler iQ system. The data obtained was analyzed using the $\triangle \mathrm{CT}$ threshold cycle method in which GAPDH was used as the normalizing gene.

Statistics. Comparisons of percent incidence for each group were performed using Kaplan-Meier log-rank analysis. Clinical macroscopic parameters, semiquantitative histopathology scores, relative cytokine message levels, and MPO activity were analyzed using either the nonparametric Mann-Whitney $U$ test or the 1-tailed Student's $t$ test for comparing means or medians between groups as indicated. $P$ values of 0.05 or less were considered significant.

\section{Acknowledgments}

We would like to thank Robert Colbert (Cincinnati Children's Hospital) for his helpful discussion and review of this manu-

script. We thank Jill Potter, Amanda Hutchinson, Maureen Shaw, and Keith Kombrinck for their excellent technical assistance. This work was supported by NIH grant AR049822 (to J.L. Degen) and by an Arthritis Investigator Award from the Arthritis Foundation (to S. Thornton).

Received for publication August 22, 2006, and accepted in revised form July 17, 2007.

Address correspondence to: Jay L. Degen, Children's Hospital Research Foundation, Developmental Biology ML7007, CHRF Room 2042, 3333 Burnet Avenue, Cincinnati, Ohio 452293039, USA. Phone: (513) 636-4679; Fax: (513) 636-4317; E-mail: degenjl@cchmc.org.

1. Feldmann, M., Brennan, F.M., and Maini, R.N. 1996. Rheumatoid arthritis. Cell. 85:307-310.

2. Arend, W.P. 1997. The pathophysiology and treatment of rheumatoid arthritis. Arthritis Rheum. 40:595-597.

3. Kollias, G., Douni, E., Kassiotis, G., and Kontoyiannis, D. 1999. The function of tumour necrosis factor and receptors in models of multi-organ inflammation, rheumatoid arthritis, multiple sclerosis, and inflammatory bowel disease. Ann. Rheum. Dis. 58(Suppl. 1):I32-I39.

4. Yamanishi, Y., and Firestein, G.S. 2001. Pathogenesis of rheumatoid arthritis: the role of synoviocytes. Rheum. Dis. Clin. North Am. 27:355-371.

5. Feldmann, M., and Maini, R.N. 1999. The role of cytokines in the pathogenesis of rheumatoid arthritis. Rheumatology. (Oxford) 38(Suppl. 2):3-7.

6. Alonzi, T., et al. 1998. Interleukin 6 is required for the development of collagen-induced arthritis. J. Exp. Med. 187:461-468.

7. Wooley, P.H., Dutcher, J., Widmer, M.B., and Gillis, S. 1993. Influence of a recombinant human soluble tumor necrosis factor receptor FC fusion protein on type II collagen-induced arthritis in mice. J. Immunol. 151:6602-6607.

8. Williams, R.O., Ghrayeb, J., Feldmann, M., and Maini, R.N. 1995. Successful therapy of collageninduced arthritis with TNF receptor-IgG fusion protein and combination with anti-CD4. Immunology. 84:433-439.

9. Zwerina, J., et al. 2004. Single and combined inhibition of tumor necrosis factor, interleukin-1, and RANKL pathways in tumor necrosis factor-induced arthritis: effects on synovial inflammation, bone erosion, and cartilage destruction. Arthritis Rheum. 50:277-290.

10. Altieri, D.C., Mannucci, P.M., and Capitanio, A.M. 1986. Binding of fibrinogen to human monocytes. J. Clin. Invest. 78:968-976.

11. Rubel, C., et al. 2002. Soluble fibrinogen modulates neutrophil functionality through the activation of an extracellular signal-regulated kinase-dependent pathway. J. Immunol. 168:3527-3535.

12. Rubel, C., et al. 2003. Fibrinogen-CD11b/CD18 interaction activates the NF-kappa B pathway and delays apoptosis in human neutrophils. Eur. J. Immunol. 33:1429-1438.

13. Ugarova, T.P., et al. 1998. Identification of a novel recognition sequence for integrin alphaM beta 2 within the gamma-chain of fibrinogen. J. Biol. Chem. 273:22519-22527.

14. Flick, M.J., et al. 2004. Leukocyte engagement of fibrin(ogen) via the integrin receptor $\alpha \mathrm{M} \beta 2 / \mathrm{Mac}-1$ is critical for host inflammatory response in vivo. J. Clin. Invest. 113:1596-1606. doi:10.1172/ JCI200420741.

15. Varisco, P.A., et al. 2000. Effect of thrombin inhibition on synovial inflammation in antigen induced arthritis. Ann. Rheum. Dis. 59:781-787.

16. Marty, I., et al. 2001. Amelioration of collageninduced arthritis by thrombin inhibition. J. Clin. Invest. 107:631-640.

17. Esmon, C.T. 2001. Role of coagulation inhibitors in inflammation. Thromb. Haemost. 86:51-56.

18. Zacharski, L.R., et al. 1992. Pathways of coagulation activation in situ in rheumatoid synovial tissue. Clin. Immunol. Immunopathol. 63:155-162.

19. Yang, Y.H., Carmeliet, P., and Hamilton, J.A. 2001. Tissue-type plasminogen activator deficiency exacerbates arthritis. J. Immunol. 167:1047-1052.

20. Holmback, K., Danton, M.J., Suh, T.T., Daugherty, C.C., and Degen, J.L. 1996. Impaired platelet aggregation and sustained bleeding in mice lacking the fibrinogen motif bound by integrin alpha IIb beta 3 . EMBO J. 15:5760-5771.

21. Keffer, J., et al. 1991. Transgenic mice expressing human tumour necrosis factor: a predictive genetic model of arthritis. EMBOJ. 10:4025-4031.

22. Suh, T.T., et al. 1995. Resolution of spontaneous bleeding events but failure of pregnancy in fibrinogen-deficient mice. Genes Dev. 9:2020-2033.

23. Szanto, S., Bardos, T., Gal, I., Glant, T.T., and Mikecz, K. 2004. Enhanced neutrophil extravasation and rapid progression of proteoglycan-induced arthritis in TSG-6-knockout mice. Arthritis Rheum. 50:3012-3022.

24. Anitua, E., Andia, I., Ardanza, B., Nurden, P., and Nurden, A.T. 2004. Autologous platelets as a source of proteins for healing and tissue regeneration. Thromb. Haemost. 91:4-15.

25. Arend, W.P., and Dayer, J.M. 1995. Inhibition of the production and effects of interleukin-1 and tumor necrosis factor alpha in rheumatoid arthritis. Arthritis Rheum. 38:151-160.

26. Gitter, B.D., Labus, J.M., Lees, S.L., and Scheetz, M.E. 1989. Characteristics of human synovial fibroblast activation by IL-1 beta and TNF alpha. Immunology. 66:196-200.

27. Gross, T.J., Leavell, K.J., and Peterson, M.W. 1997. CD11b/CD18 mediates the neutrophil chemotactic activity of fibrin degradation product D domain. Thromb. Haemost. 77:894-900.

28. Ding, Z.M., et al. 1999. Relative contribution of LFA- 1 and Mac-1 to neutrophil adhesion and migration. J. Immunol. 163:5029-5038.

29. Mizgerd, J.P., et al. 1997. Neutrophil emigration in the skin, lungs, and peritoneum: different requirements for CD11/CD18 revealed by CD18-deficient mice. J. Exp. Med. 186:1357-1364.

30. Prince, J.E., et al. 2001. The differential roles of LFA-1 and Mac- 1 in host defense against systemic infection with Streptococcus pneumoniae. J. Immunol. 166:7362-7369.

31. Lu, H., et al. 1997. LFA-1 is sufficient in mediating neutrophil emigration in Mac-1-deficient mice. J. Clin. Invest. 99:1340-1350.
32. Lawlor, K.E., Wong, P.K., Campbell, I.K., van Rooijen, N., and Wicks, I.P. 2005. Acute CD4+ T lymphocyte-dependent interleukin-1-driven arthritis selectively requires interleukin-2 and interleukin-4, joint macrophages, granulocyte-macrophage colony-stimulating factor, interleukin-6, and leukemia inhibitory factor. Arthritis Rheum. 52:3749-3754.

33. Tanaka, D., Kagari, T., Doi, H., and Shimozato, T. 2006. Essential role of neutrophils in anti-type II collagen antibody and lipopolysaccharide-induced arthritis. Immunology. 119:195-202.

34. Vossenaar, E.R., Zendman, A.J., and Van Venrooij, W.J. 2004. Citrullination, a possible functional link between susceptibility genes and rheumatoid arthritis. Arthritis Res. Ther. 6:1-5.

35. Masson-Bessiere, C., et al. 2001. The major synovial targets of the rheumatoid arthritis-specific antifilaggrin autoantibodies are deiminated forms of the alpha- and beta-chains of fibrin. J. Immunol. 166:4177-4184.

36. Aho, K., Palusuo, T., and Kurki, P. 1994. Marker antibodies of rheumatoid arthritis: diagnostic and pathogenetic implications. Semin. Arthritis Rheum. 23:379-387.

37. Vincent, C., et al. 1989. High diagnostic value in rheumatoid arthritis of antibodies to the stratum corneum of rat oesophagus epithelium, socalled 'antikeratin antibodies'. Ann. Rheum. Dis. 48:712-722.

38. Kuhn, K.A., et al. 2006. Antibodies against citrullinated proteins enhance tissue injury in experimental autoimmune arthritis. J. Clin. Invest. 116:961-973. doi:10.1172/JCI25422.

39. Probert, L., Plows, D., Kontogeorgos, G., and Kollias, G. 1995. The type I interleukin-1 receptor acts in series with tumor necrosis factor (TNF) to induce arthritis in TNF-transgenic mice. Eur. J. Immunol. 25:1794-1797.

40. Busso, N., et al. 1998. Exacerbation of antigeninduced arthritis in urokinase-deficient mice. J. Clin. Invest. 102:41-50.

41. Cook, A.D., Braine, E.L., Campbell, I.K., and Hamilton, J.A. 2002. Differing roles for urokinase and tissue-type plasminogen activator in collageninduced arthritis. Am. J. Pathol. 160:917-926.

42. Li, J., et al. 2005. The plasminogen activator/plasmin system is essential for development of the joint inflammatory phase of collagen type II-induced arthritis. Am. J. Pathol. 166:783-792.

43. Thornton, S., Boivin, G.P., Kim, K.N., Finkelman, F.D., and Hirsch, R. 2000. Heterogeneous effects of IL-2 on collagen-induced arthritis. J. Immunol. 165:1557-1563.

44. Flick, M.J., Du, X., and Degen, J.L. 2004. Fibrin(ogen)-alpha M beta 2 interactions regulate leukocyte function and innate immunity in vivo. Exp. Biol. Med. (Maywood). 229:1105-1110. 